\title{
YKL-40 in the brain and cerebrospinal fluid of neurodegenerative dementias
}

Franc Llorens ${ }^{1,2^{*}}$, Katrin Thüne ${ }^{1,3}$, Waqas Tahir ${ }^{2}$, Eirini Kanata ${ }^{4}$, Daniela Diaz-Lucena ${ }^{1}$, Konstantinos Xanthopoulos ${ }^{4,12}$, Eleni Kovatsi ${ }^{4}$, Catharina Pleschka ${ }^{5}$, Paula Garcia-Esparcia ${ }^{1,6}$, Matthias Schmitz ${ }^{2,3}$, Duru Ozbay ${ }^{2}$, Susana Correia ${ }^{2}$, Ângela Correia ${ }^{2}$, Ira Milosevic ${ }^{7}$, Olivier Andréoletti ${ }^{8}$, Natalia Fernández-Borges ${ }^{9}$, Ina M. Vorberg ${ }^{5}$, Markus Glatzel ${ }^{10}$, Theodoros Sklaviadis ${ }^{4}$, Juan Maria Torres ${ }^{9}$, Susanne Krasemann ${ }^{10}$, Raquel Sánchez-Valle ${ }^{11}$, Isidro Ferrer ${ }^{1,6+}$ and Inga Zerr ${ }^{2,3+}$

\begin{abstract}
Background: YKL-40 (also known as Chitinase 3-like 1) is a glycoprotein produced by inflammatory, cancer and stem cells. Its physiological role is not completely understood but YKL-40 is elevated in the brain and cerebrospinal fluid (CSF) in several neurological and neurodegenerative diseases associated with inflammatory processes. Yet the precise characterization of YKL-40 in dementia cases is missing.

Methods: In the present study, we comparatively analysed YKL-40 levels in the brain and CSF samples from neurodegenerative dementias of different aetiologies characterized by the presence of cortical pathology and disease-specific neuroinflammatory signatures.

Results: YKL-40 was normally expressed in fibrillar astrocytes in the white matter. Additionally YKL-40 was highly and widely expressed in reactive protoplasmic cortical and perivascular astrocytes, and fibrillar astrocytes in sporadic Creutzfeldt-Jakob disease (sCJD). Elevated YKL-40 levels were also detected in Alzheimer's disease (AD) but not in dementia with Lewy bodies (DLB). In AD, YKL-40-positive astrocytes were commonly found in clusters, often around $\beta$-amyloid plaques, and surrounding vessels with $\beta$-amyloid angiopathy; they were also distributed randomly in the cerebral cortex and white matter. YKL-40 overexpression appeared as a pre-clinical event as demonstrated in experimental models of prion diseases and AD pathology.

CSF YKL-40 levels were measured in a cohort of 288 individuals, including neurological controls (NC) and patients diagnosed with different types of dementia. Compared to NC, increased YKL-40 levels were detected in SCJD $(p<0.001, A U C=0.92)$ and $\mathrm{AD}(p<0.001, \mathrm{AUC}=0.77)$ but not in vascular dementia $\mathrm{VaD})(p>0.05, \mathrm{AUC}=0.71)$ or in DLB/Parkinson's disease dementia (PDD) $(p>0.05$, AUC $=0.70)$. Further, two independent patient cohorts were used to validate the increased CSF YKL-40 levels in SCJD. Additionally, increased YKL-40 levels were found in genetic prion diseases associated with the PRNP-D178N (Fatal Familial Insomnia) and PRNP-E200K mutations.
\end{abstract}

Conclusions: Our results unequivocally demonstrate that in neurodegenerative dementias, YKL-40 is a disease-specific marker of neuroinflammation showing its highest levels in prion diseases. Therefore, YKL-40 quantification might have a potential for application in the evaluation of therapeutic intervention in dementias with a neuroinflammatory component.

Keywords: Chitinase 3-like 1, YKL-40, Neuroinflammation, Cerebrospinal fluid, Neurodegenerative dementias, Brain

\footnotetext{
* Correspondence: franc.llorens@gmail.com

${ }^{\dagger}$ Equal contributors

'Network Center for Biomedical Research in Neurodegenerative Diseases

(CIBERNED), Institute Carlos III, Ministry of Health, Feixa Llarga s/n, 08907

L'Hospitalet de Llobregat, Barcelona, Spain

2Department of Neurology, University Medical School, Göttingen, Germany

Full list of author information is available at the end of the article
} 


\section{Background}

YKL-40 is a glycoprotein secreted by various cell-types including macrophages, chondrocytes, vascular smooth muscle cells and some types of cancer cells [1-4]. In normal adult human tissue higher YKL-40 expression is observed in cells with high cellular activity [5]. Although its precise function is not well understood, it is hypothesized to be involved in tissue remodelling during inflammation and in angiogenic processes mediating infiltration, differentiation and maturation of macrophages, and it is therefore considered a marker of inflammation and endothelial dysfunction [6-9]. Elevated YKL-40 is reported in many pathological conditions such as cancer, diabetes mellitus, cardiovascular disorders and inflammatory diseases of different aetiologies including, among others: bacterial infections, osteoarthritis, hepatic fibrosis, ulcerative colitis, Crohn's disease, rheumatoid arthritis, asthma, chronic obstructive pulmonary disease and liver cirrhosis [4, 10-14].

In the brain, YKL-40 is upregulated in several neurological disorders such as stroke, lentiviral encephalitis, traumatic brain injury, amyotrophic lateral sclerosis, multiple sclerosis and Alzheimer's disease (AD) [15-18]. Although YKL-40 was initially associated with the macrophage lineage [19-21], evidence suggests that during neuroinflammatory processes, its expression is abundant in reactive astrocytes and residual in microglial cells $[16,22]$. Indeed, astrocytic YKL-40 expression has been reported in both acute and chronic neurological conditions [16] and in close vicinity to amyloid plaques and neurofibrillary tangles in AD [23]. In CSF, YKL-40 is elevated in several acute and chronic neuroinflammatory conditions [16] and in preclinical and prodromal $\mathrm{AD} /$ mild cognitive impairment (MCI) [23-26], which is in agreement with the potential role of astrocytosis in early AD pathogenesis [27]. Interestingly, YKL-40 is associated with cerebral morphometric patterns different from those linked to phospho-tau (p-tau)-related neurodegeneration [28], indicating a dissociation between astrocytic activation and p-tau pathology in early AD. Increased CSF YKL-40 levels have been reported in multiple AD cohorts [29-34], and in frontotemporal dementia (FTD) cases [34-36] but not in vascular dementia $(\mathrm{VaD})$ and in $\mathrm{DLB} / \mathrm{PDD}[32,35]$, indicating that its deregulation is associated with disease-specific alterations and further suggesting that elevated CSF YKL-40 levels is not a specific signature for AD pathogenesis. However, conflicting data regarding non-AD dementias has been reported $[35,37]$. Moreover, absence of studies reporting YKL-40 levels in prion diseases, which present the highest neuroinflammatory component among all dementia conditions [38], impedes establishment of the precise accuracy of YKL-40 quantification in the differential diagnostic context of neurodegenerative dementias. Further, brain
YKL-40 expression patterns in different dementia types, and the potential correlation between brain and CSF levels is largely unknown, indicating that more research regarding YKL-40 expression patterns is required. Based on the data presented before, YKL-40 is in the spot light of biomarker research.

In this study, we investigated the sources of YKL-40 in the brain and evaluated its levels in the CSF in different types of neurodegenerative dementias, allowing us to determine the accuracy of YKL-40 quantification in the differential diagnostic context. Our data revealed that YKL-40 expression, while residual in the white matter of control cases, was highly increased in reactive protoplasmic and perivascular astrocytes in $\mathrm{SCJD}$ and $\mathrm{AD}$ cases. Among the different disease groups studied, highest YKL-40 levels in brain and CSF were observed in SCJD cases, followed by AD and DLB.

\section{Methods \\ Reagents and antibodies}

Detection of human YKL-40 by western blot was performed using the Human Chitinase 3-like 1 Antibody AF2599 (R\&D Biosystems). Detection of murine YKL-40 by western blot, and human and murine YKL.40 by immunohistochemistry and immunofluorescence was performed using the Chitinase 3-like 1 antibody PA5-43746 (Thermo Fisher).

Anti-GAPDH 9484, anti- $\beta$-actin 8226 and antivimentin 92547 antibodies were from Abcam. Mouse anti-GFAP MAB360 was from Millipore. Rabbit AntiGFAP Z0334 and anti-amyloid beta ( $\beta$-amyloid) M0872 were from Dako. Anti-PrP(12F10) A03221 was from SPI Bio. Thioflavin was from Sigma. Chimeric recombinant prion protein $(\mathrm{PrP})$, composed of the Syrian hamster residues 23-137 followed by sheep residues 141-234 (of the R154 Q171 polymorphism), was from Thermo Fisher.

\section{Demographics and human cases}

Brain tissue was obtained from the Institute of Neuropathology Brain Bank (HUB-ICO-IDIBELL Biobank) and the Biobank of Hospital Clinic-IDIBAPS following relevant guidelines of both Spanish legislation and the local ethics committee. Neuropathological examination and characterization were carried out on paraffin-embedded samples in every case. Detailed information on neuropathology and inflammatory profiling of the SCJD and AD cohorts has been previously reported [39-42]. sCJD MM1 and VV2 cases were selected due to their higher prevalence, but different clinical phenotypes [43]. The presence of infectious, metabolic and neoplastic diseases was ruled out in control samples. No correlation between postmortem delay or sample storage time and levels of proteins and mRNA analysed was observed. A full summary of the cases is available in Additional file 1. 
In order to establish the potential diagnostic utility of CSF YKL-40 measurement in the context of differential diagnosis of neurodegenerative dementias, we utilized samples from three independent cohorts: i) cases recruited at the Clinical Dementia Center at the University Medical Center Göttingen (Germany) (cohort 1), ii) cases recruited at the Hospital Clinic de Barcelona (Spain) (cohort 2) and iii) cases recruited at hospitals of northern Greece that were further processed at the Laboratory of Pharmacology (School of Pharmacy, Aristotle University of Thessaloniki), as part of additional molecular analysis to enhance disease diagnosis (cohort 3). Lumbar puncture was performed for diagnostic purposes followed by analysis of CSF standard parameters. YKL-40, tau, $14-3-3$ and A $\beta 42$ were analysed at the point of the first diagnostic work-up. In cohort 1 , the neurological control group was composed of patients with either clinically or pathologically defined alternative diagnoses $[44,45]$. This group included cases of psychiatric disorders, ischemic stroke, epilepsy, autoimmune diseases, alcohol abuse, headache, vertigo, pain syndromes, acute hypoxia and non-related neurological conditions. Control cases did not present biomarker profiles indicating the presence of a neurodegenerative disease. Diagnosis of $\mathrm{AD}$ was based on the International Classification of Diseases, Tenth Revision (ICD-10) definition for AD (F.00-G.30). Rapidly progressive Alzheimer's disease (rpAD) was defined by a velocity of cognitive decline $>6 \mathrm{pts} / \mathrm{yr}$. on the Mini Mental Status Examination scale [46]. Velocity of decline was calculated using linear regression (least square method) in accordance with Villemagne et al. [47]. The diagnosis of DLB was based on the criteria of McKeith [17] and PDD as dementia in patients with $\mathrm{PD}$. $\mathrm{VaD}$ diagnosis was based on ICD 10 definition (F01) and NINDS-AIREN criteria [48]. In cohort 2, controls were neurologically healthy individuals with no neurological clinical diagnosis and normal neuropsychological assessment. AD patients were diagnosed with probable $\mathrm{AD}$ using the National Institute of Neurological and Communicative Disorders and Stroke/Alzheimer's Disease and Related Disorders Association criteria [49] and displayed the typical CSF biomarker profile, characteristic of AD (low levels of amyloid beta 1-42 (A $\beta 42)$ and elevated $\mathrm{t}$-tau and $\mathrm{p}$-tau levels). In cohort 3 , controls were composed of subjects with cognitive impairment/dementia (of unknown kind) with excluded prion diagnosis. sCJD cases were classified as definite cases by neuropathological examinations or as probable cases according to diagnostic consensus criteria [50, 51]. Genetic prion cases were confirmed by genetic testing (PRNP D178N and E200K mutations). Additional file 2 provides information on demographics and CSF biomarkers results.

\section{Experimental mouse models}

As a sCJD MM1 mouse model, the tg340 mouse line expressing about 4-fold level of human PrP M129 on a mouse PrP null background [52] was used. Control or SCJD MM1 brain tissues were inoculated as $10 \%(w / v)$ homogenates in 6-10 week-old mice in the right parietal lobe using a 25-gauge disposable hypodermic needle. Mice were observed daily and the neurological status was assessed weekly. The animals were sacrificed at presymptomatic (pre-clinical: 120 day post inoculation (dpi) and symptomatic (early clinical: $160 \mathrm{dpi}$ and late clinical: 183 dpi) stages. Additionally, sCJD MM1 inoculum dilutions were performed to study prolonged disease times; animals were sacrificed at $210 \mathrm{dpi}$ (10-1 dilution). Part of the brain was fixed by immersion in $10 \%$ buffered formalin to quantify spongiform degeneration and perform immunohistological analyses. The remaining brain was snap frozen and stored at $-80{ }^{\circ} \mathrm{C}$ for protein and RNA studies. The 5xFAD mouse line harbouring the APP KM670/671NL (Swedish), APP I716V (Florida), APP V717I (London), PSEN1 M146 L (A\&gt;C), PSEN1 L286 V mutations was used as a model of AD pathogenesis. This widely-used model recapitulates several ADrelated phenotypes displaying severe amyloid pathology and gliosis at about 2-3 months, cognitive alterations at 4-5 months and synaptic and neuronal loss at 9 of age $[53,54]$. For scrapie infections with prion strains RML (immunohistochemical data) and $22 \mathrm{~L}$ (qPCR data), all animal experiments were in strict accordance with the principles of laboratory animal care $(\mathrm{NIH}$ publication No. 86-23, revised 1985) and the recommendations in the Guide for the Care and Use of Laboratory Animals of the German Animal Welfare Act on protection of animals. Animals were maintained under specific pathogenfree conditions. Mice were anesthetized with Ketamine/ Xylazin hydrochloride prior to inoculation. Eight weeks old mice were inoculated intracerebrally (i.c.) with RML 5.0-prions (immunohistochemical data) and $22 \mathrm{~L}$ prions (qPCR data). Animals were checked daily and were taken at indicated time points or were allowed to progress to clinical prion disease (presenting with slowness, ataxia, weight loss, trembling and ungroomed fur). For scrapie infections with RML strain (qPCR data), all experiments were performed in the animal facility of the Neurological Department, AHEPA University Hospital. Approximately 8-12 weeks old mice were inoculated. Infectious material corresponding to $100 \mu \mathrm{l}$ of $1 \%$ brain homogenate from terminally ill RML-infected C57Bl/6 mice $(104.5 \times$ LD50) was intraperitoneally administered in fourteen animals; ten age-matched unchallenged mice were used as controls. RML-infected $\mathrm{C} 57 \mathrm{Bl} / 6$ mice developed the first clinical symptoms, corresponding to abnormal gait, at approximately 130 days post inoculation. More severe symptoms, including loss of muscle tone, 
hind limb weakness, ataxia, weight loss and ruffled coat, appeared at approximately 160 days post inoculation. Whole brain was harvested from three animals per group at pre-clinical $(120 \mathrm{dpi})$ and end-point clinical (180 dpi) disease stages.

\section{Organotypic slice cultures}

Organotypic cerebellar slice preparations from postnatal day 9-13 (P9-13) pups of C57BL/6)Rj mice, further infection with $22 \mathrm{~L}$ scrapie strain, sample collection and detection of PK-resistant Prion Protein (PrPres) were performed as described previously [55].

\section{CSF analysis}

Total tau was measured using INNOTEST ${ }^{\mathrm{Tx}}$ hTAU Ag; (Fujirebio). Levels of amyloid $\beta 1-42$ (A $\beta 42)$ were measured with an ELISA kit (INNOTEST ${ }^{\mathrm{m}}$ AMYLOID (1-42); Fujirebio). YKL-40 was measured using the MicroVue YKL-40 EIA ELISA kit from Quidel following the manufacturer's instructions. Protein 14-3-3 was detected with western blot as previously described [56].

\section{RNA purification and RT-qPCR}

RNA from different human and mouse brain regions was purified using the miRVANA RNA isolation kit following the manufacturer's protocol. For human samples, regions utilized for RNA extraction and corresponding age and gender information is summarized in Additional file 1. RNA was extracted from the following murine brain regions: tg340MM1-CJDMM1: cerebral cortex, RML scrapie: whole brain, 22 L scrapie: cerebral cortex and cerebellum. RNAs were treated with DNase Set (Qiagen) to eliminate genomic DNA contamination. Sample concentration was measured using a NanoDrop 2000 spectrophotometer (Thermo Scientific) and RNA integrity was assessed based on the RNA Integrity Number (RIN value) measured by an Agilent 2100 Bioanalyzer (Agilent). RNA samples were retrotranscribed using the High Capacity cDNA Archive kit (Applied Biosystems). Quantitative PCR assays were performed in duplicates on cDNA samples, in a LightCycler ${ }^{\circ} 480$ System (Roche). The reactions were set using 20xTaqMan Gene Expression Assays and 2xTaqMan Universal PCR Master Mix (Applied Biosystems) and conducted under the following conditions: $50{ }^{\circ} \mathrm{C}$ for $2 \mathrm{~min}, 95{ }^{\circ} \mathrm{C}$ for $10 \mathrm{~min}, 40$ cycles at $95^{\circ} \mathrm{C}$ for $15 \mathrm{~s}$ and $60{ }^{\circ} \mathrm{C}$ for $1 \mathrm{~min}$. Fold changes were estimated using the $2-\Delta \Delta C \mathrm{C}$ equation. Fold changes in mRNA expression were determined relative to the control cases after normalization with housekeeping genes; statistical significances were calculated using GraphPad Prism 6.01. As housekeeping genes, GAPDH and GUSB as well as Gusb and Hprt were used as in human and mouse samples respectively. The Taqman probes used were: CHI3L1 (YKL-40) human:
Hs01072228_m1, GFAP human: Hs00909240_m1, AIF1 (IBA-1) human: Hs00741549_g1, GAPDH human: Hs039 29097_g1, GUSB human: Hs00939627_m1, Chi3l1 (YKL40) mouse: Mm00801477_m1, Hprt mouse: Mm0302 4075_m1, GusB mouse: Mm00446958_g1.

\section{Western blotting}

Human and mouse tissues were lysed in lysis buffer con-

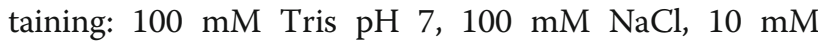
EDTA, $0.5 \%$ NP-40 and $0.5 \%$ sodium deoxycolate plus protease and phosphatase inhibitors (Sigma). Samples were centrifuged $\left(14,000 \times \mathrm{g}\right.$ for $20 \mathrm{~min}$ at $\left.4{ }^{\circ} \mathrm{C}\right)$ and supernatants were quantified for protein concentration using the Bradford method (Biorad). Samples were mixed with SDS-PAGE sample buffer, heated to $95{ }^{\circ} \mathrm{C}$ for $10 \mathrm{~min}$, and resolved by SDS-PAGE analysis. Gels were transferred onto PVDF membranes, blocked and incubated with anti-YKL-40 antibodies (1:1000 dilution) as indicated above. Following incubation with HRPconjugates, species-specific secondary antibody, the membranes were processed for specific immunodetection using the ECL reagent. GAPDH and $\beta$-actin antibodies at 1:5000 dilutions were used as loading controls. Fold changes (in arbitrary units) were determined from densitometric analysis relative to the control cases.

\section{Immunohistochemistry and immunofluorescence}

The immunohistochemical study was performed on $4-\mu \mathrm{m}$ thick dewaxed brain sections. Endogenous peroxidases were blocked with peroxidase (Dako, Glostrup) followed by $10 \%$ normal goat serum. Following incubation with the anti-YKL-40 antibody (diluted 1:200) at room temperature overnight, the sections were incubated with EnVision1 system peroxidase (Dako) for $15 \mathrm{~min}$ at room temperature. The peroxidase reaction was visualized with diaminobenzidine (DAB) and $\mathrm{H}_{2} \mathrm{O}_{2}$. Primary antibody was omitted in some sections as immunostaining control and no signal was detected after incubation with the secondary antibody. Sections were counterstained with haematoxylin. For immunofluorescence, $4 \mu \mathrm{m}$ thick dewaxed sections were stained with a saturated solution of Sudan black B (Merck, Germany) for $15 \mathrm{~min}$ to block the autofluorescence of lipofuscin granules present in cell bodies, and then rinsed in $70 \%$ ethanol and washed in distilled water. The sections were boiled in citrate buffer to enhance antigenicity, blocked for $30 \mathrm{~min}$ at room temperature with $10 \%$ fetal bovine serum diluted in phosphate buffered saline, and incubated at $4{ }^{\circ} \mathrm{C}$ overnight with primary antibodies. Double-labelling immunofluorescence was performed using a combination of YKL-40 (1:100) and GFAP (1:200 or 1:400) antibodies or $\operatorname{PrP}(12 \mathrm{~F} 10)$ (1:200) and either A $\beta$ (1:50), YKL40 (1:100) or GFAP (1:400) antibodies. After washing, the sections were incubated with Alexa (Molecular Probes) fluorescence 
secondary antibodies (Alexa-Fluor 488 and 555 conjugated secondary antibodies). Nuclei were stained with DRAQ5 $5^{\mathrm{mm}}$ (Biostatus Ltd., Shepshed, UK). After washing, the sections were mounted in Immuno-Fluore mounting medium (ICN Biomedicals, Irvine, CA), sealed and dried overnight. Confocal images were acquired using a microscope Leica DMIRE2 and Leica confocal software. Z-Stack image interval was $0.5 \mu \mathrm{m}$. Single plane images for quantification were obtained by Nikon Eclipse E800 microscope with ProgRes ${ }^{\circ}$ CapturePro 2.7.7 software (JENOPTIK). Quantification of YKL-40 and/or GFAP positive cells was performed manually using Fiji ImageJ software in 13-15 areas per section in 3 different patients.

Immunofluorescence of frozen brain sections in $5 x F A D$ mice was carried out as described [57]. For the analysis of human samples, two to four cases per diagnostic group were used and three to four sections from every case were analysed. For immunohistochemical analysis upon prion infection, tissues were fixed with $4 \%$ buffered formalin, prion infected samples were inactivated by immersion in $98 \%$ formic acid for one hour, post fixed in $4 \%$ buffered formalin overnight and processed for paraffin embedding. Sections $(2 \mu \mathrm{m})$ were subjected to staining with the CHI3L1 antibody (YKL-40, 1:100) using a Ventana Benchmark XT (Ventana, Tuscon, Arizona, USA). Sections were incubated with primary antibody for $1 \mathrm{~h}$, anti-rabbit Histofine Simple Stain MAX PO Universal immunoperoxidase polymer (Nichirei Biosciences, Wedel, Germany) was used as secondary antibody. Detection of secondary antibodies and counter staining was performed with an ultraview universal DAB detection kit from Ventana (Ventana, Tuscon, Arizona, USA). Representative images were taken with a Leica DMD108 digital microscope.

\section{Elisa}

Detection of murine YKL-40 in tissue homogenates was performed using the Mouse Chitinase 3-like 1/YKL-40 PicoKine $^{\text {tw }}$ ELISA Kit (Bosterbio) following the manufacturer's instructions. Inter- and intra assay coefficients of variation were below $15 \%$. Dilution linearity tested from neat CSF to 1:8 dilution factor. Percent linearity was 85$112 \%$. For the assays, CSF was diluted 1:3.

\section{Real-time quaking induced conversion (RT-QulC)}

RT-QuIC was performed as described previously $[58,59]$ with minor modifications. Briefly, $85 \mu \mathrm{l}$ of reaction buffer containing recombinant $\operatorname{PrP}(10 \mu \mathrm{g})$ was mixed with $15 \mu \mathrm{l}$ of a 1:100 dilution of $10 \% w / v$ brain homogenates. Tissues for RT-QuIC analysis were lysed in PBS $0.1 \%$ SDS and clarified by centrifugation for $10 \mathrm{~min}$ at $10000 \times$ g. Reactions were set at a final volume of $100 \mu \mathrm{l}$ and placed in 96-well black optical bottom plates (Fisher Scientific). Each sample was run in triplicate. Plates were sealed and incubated in a FLUO Star OPTIMA plate reader (BMG Labtech Ortenberg, GE) at $42{ }^{\circ} \mathrm{C}$ for $80 \mathrm{~h}$ with intermittent shaking cycles, consisting of $1 \mathrm{~min}$ double orbital shaking at the highest speed $(600 \mathrm{rpm})$ followed by a 1 min break.

\section{Statistical analysis}

For two-group comparisons the Mann-Whitney test (non-parametric distribution) and unpaired t-test (parametric distribution) were used. In multiple comparisons, the Kruskal-Wallis test was used. Dunn's multiple comparison test was used for post-hoc analysis. Values of $p<0.05$ were considered significant. Pearson (parametric distribution) and Spearman (non-parametric distribution) tests were used to detect correlations between biomarkers, demographic data and mRNA expression levels. Two-way ANOVA followed by Bonferroni's posthoc was used in the quantification YKL-40 and GFAP positive cells. Receiver operating characteristic (ROC) curves and derived area under the curve (AUC; AUC $=0.5$ no discrimination and $\mathrm{AUC}=1$ perfect discrimination) were calculated. The best cut-off value, sensitivity and specificity were estimated based on the Youden index [60] (point on an ROC curve providing the best balance of both sensitivity and specificity). ROC curves and statistical analyses were performed using the Graph Pad Prism 6.01 software.

\section{Genetic testing}

For detection of a prion disease-associated mutation, genetic testing was performed on genomic DNA isolated from blood or brain tissue [61]. Informed consent for the prion protein gene (PRNP) analysis was obtained from each patient or legal guardian.

\section{Results}

\section{YKL-40 expression in SCJD patients}

Expression of YKL-40 was initially analysed in SCJD as this neurodegenerative dementia presents the highest neuroinflammatory profile, including massive gliosis [39] and regulation of inflammatory mediators [38] compared to other neurodegenerative dementias [38, 40]. mRNA analysis showed that YKL-40 was highly increased in the frontal cortex (FC) and cerebellum (CB) of sCJD subtypes MM1 and VV2 $(p<0.001)$. No statistically significant differences were observed between SCJD subtypes in the two regions studied. Mean fold change values for controls versus SCJD comparisons were 6.1 in the FC and 11.5 in the CB (Fig. 1a). YKL-40 levels were normalized with GAPDH, and similar results were obtained for GUSB normalization (Additional file 3A). In agreement with mRNA data, YKL-40 protein levels were increased in the $\mathrm{FC}$ and $\mathrm{CB}$ of sCJD subtypes MM1 and VV2 $(p<0.001)$. Mean fold change values for controls versus 


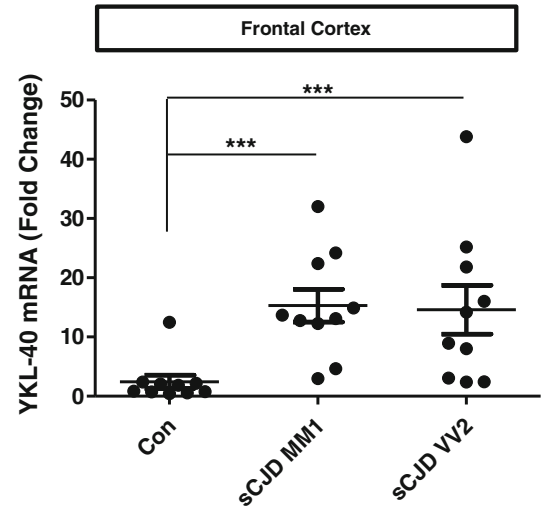

b
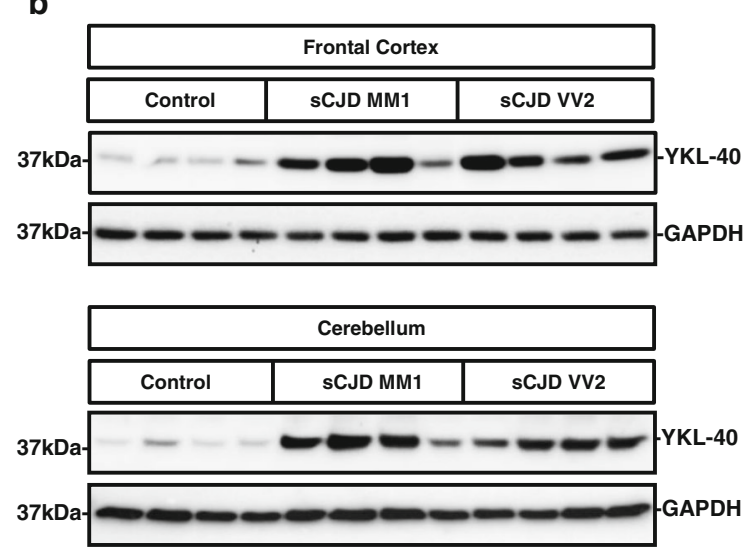
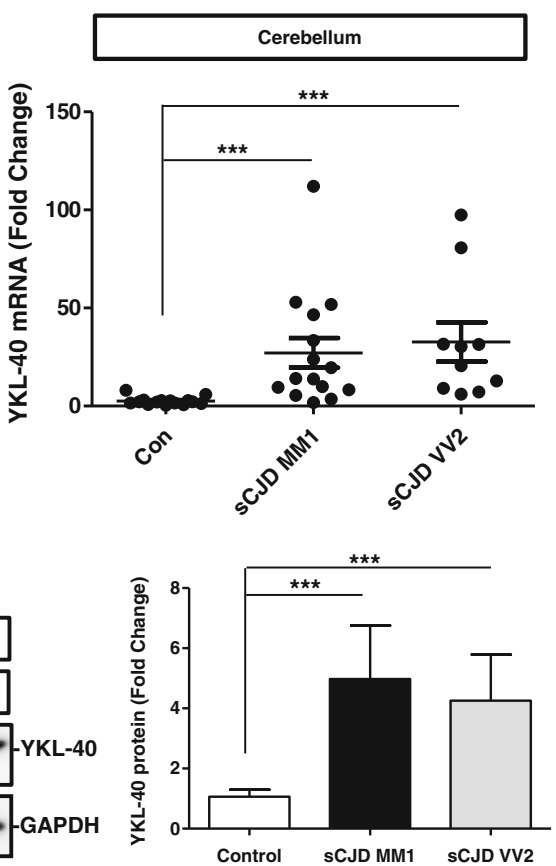

$\star \star \star *$

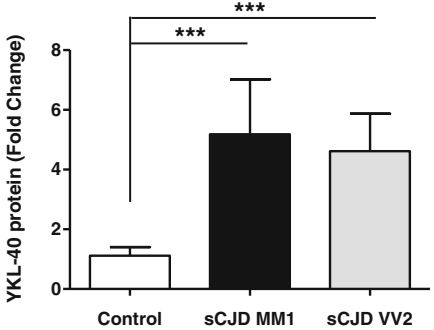

Fig. 1 YKL-40 expression in the brain tissue of sCJD and related mouse model. a RT-qPCR analysis of YKL-40 in the frontal cortex (left panel) and cerebellum (right panel) of control, sCJD MM1 and SCJD W2 samples. GAPDH was used for normalization. $\mathbf{b}$ Western blot analysis of YKL-40 in the frontal cortex (upper panel) and cerebellum (bottom panel) of control, sCJD MM1 and SCJD W2 samples. For normalization GAPDH was used. Graphical representation of Western Blot data acquired from the analysis of eight samples per group. Fold changes in the expression of mRNA and protein were determined relative to the control cases. Kruskal-Wallis and Dunn's post-hoc tests were used to determine statistical differences. ${ }^{* * *} p<0.001$

sCJD comparisons were 4.5 in the $\mathrm{FC}$ and 4.6 in the CB (Fig. 1b).

Further, immunohistochemistry was used for the study of YKL-40 expression and distribution in control and sCJD brains. YKL-40 immunoreactivity in normal brain was restricted to fibrillar astrocytes (stellate astrocytes with long processes) in the white matter and was increased in both the cerebral cortex and white matter of sCJD brain tissue sections compared to controls (Fig. 2a). YKL-40 was localized predominately in reactive protoplasmic and perivascular astrocytes, in addition to increased signal in fibrillar astrocytes of the white matter. Increased YKL-40 immunoreactivity was also detected in the Bergmann glia in the $\mathrm{CB}$ of sCJD cases (Fig. 2b). No apparent correlation was observed between PrPSc amorphous deposits in the CB in VV2 and YKL-40positive astrocytes (data not shown). Specific expression of YKL-40 in astrocytes was confirmed by a positive co-localization between YKL-40 and GFAP in FC (Fig. 2c). Interestingly, double PrP-GFAP and PrP-YKL40 immunostaining in the FC of SCJD revealed the presence of astrocytic processes within the PrP plaque-like deposits (Additional file 4A and 4B).

Additionally, we found a positive correlation between mRNA levels of YKL-40 and the astrocytic marker GFAP $(p<0.01)$ in the FC of SCJD cases, while no correlation was observed with the microglial marker AIF1 $(p>0.05)$ (Fig. 2d). As positive but moderate correlation between GFAP and YKL-40 expression levels were observed, the association between YKL-40+ and GFAP+ astrocytes was further explored. Quantification of double-labelled GFAP and YKL-40 immunoreactive cells showed co-localization in a majority of cells $($ GFAP+/YKL-40+ $=251 \pm 8$ cells $/ \mathrm{mm} 2)$ whereas the number of cells showing only GFAP staining or only YKL-40 staining was significantly smaller $(44 \pm 15$ 


\section{a}

\begin{tabular}{|c|}
\hline Control \\
\hline $\mathrm{x} 200$ \\
\hline
\end{tabular}
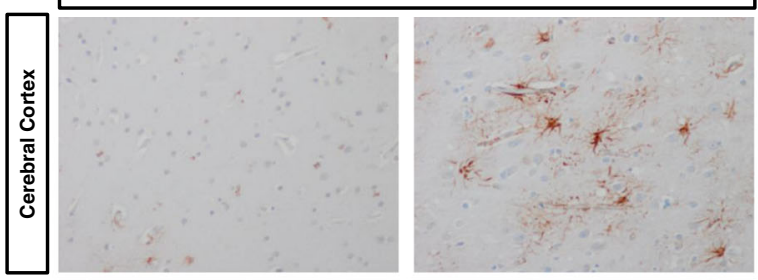

x400
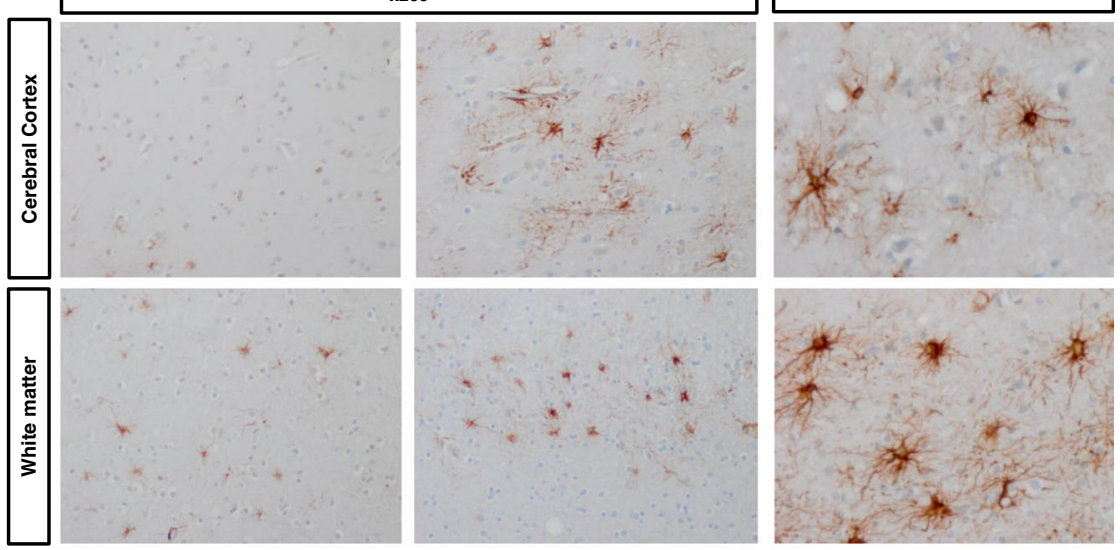

b
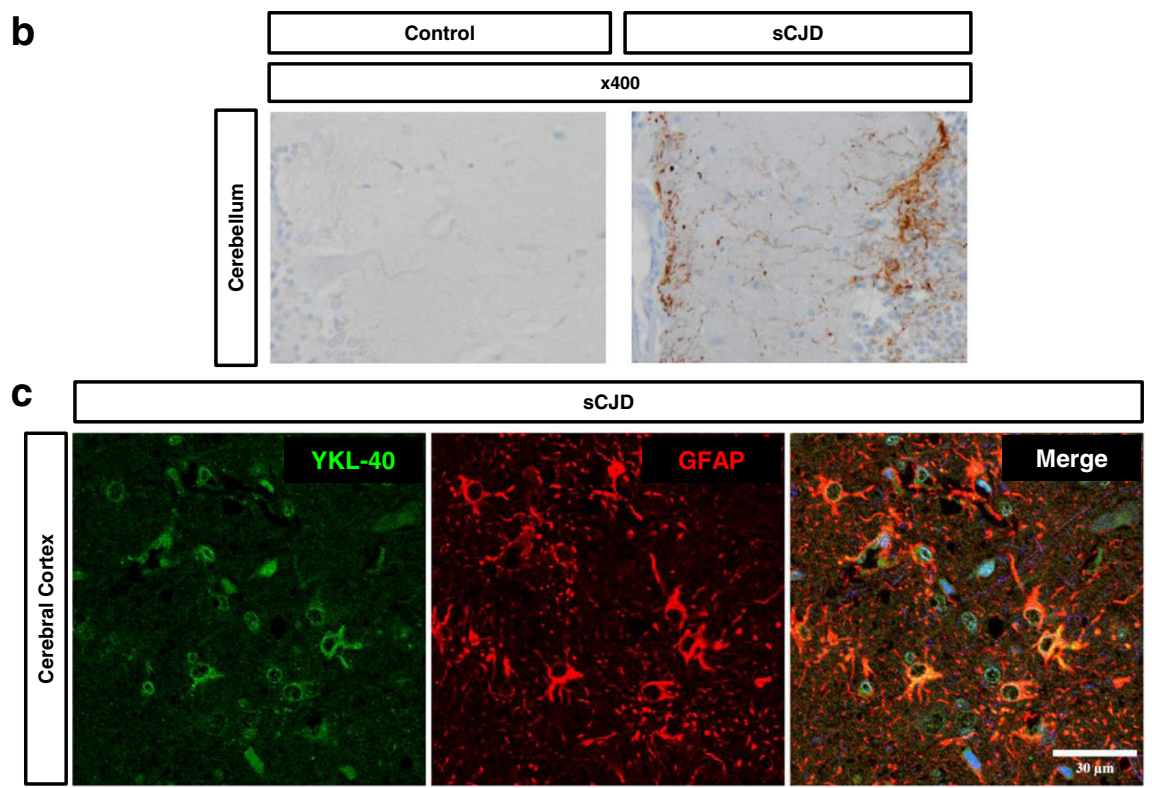

d

Frontal Cortex - sCJD
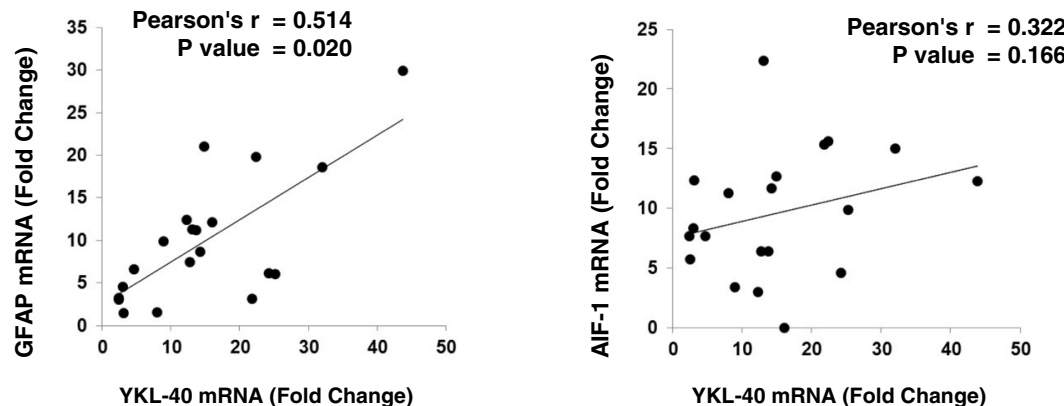

Fig. 2 YKL-40 expression in SCJD brain tissue. a Immunohistochemical analysis for detection of YKL-40 in the cerebral cortex and white matter of control and SCJD cases. $\mathbf{b}$ Immunohistochemical analysis of YKL-40 in the cerebellum of control and SCJD cases. Brown staining corresponds to YKL-40 staining and light blue to haematoxylin counterstaining. c Immunofluorescence analysis of YKL-40 (green) and GFAP (red) in cerebral cortex region of SCJD. Nuclei were stained with DAPI (blue). $\mathbf{d}$ Correlation between the expression levels of YKL-40 and GFAP mRNA (left panel) or AIF-1 mRNA (right panel) in the frontal cortex of sCJD cases. Normalization was performed using GAPDH. Pearson test was used to determine the correlations between mRNA expression levels 


\section{a}

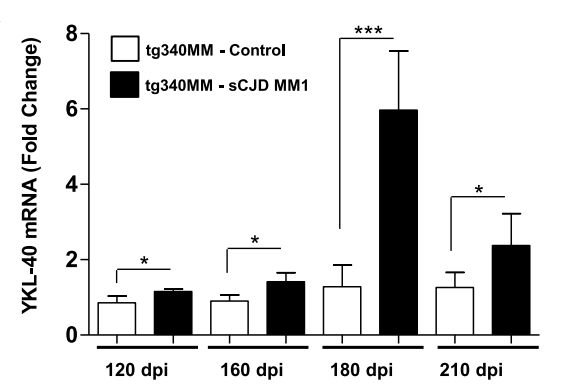

C
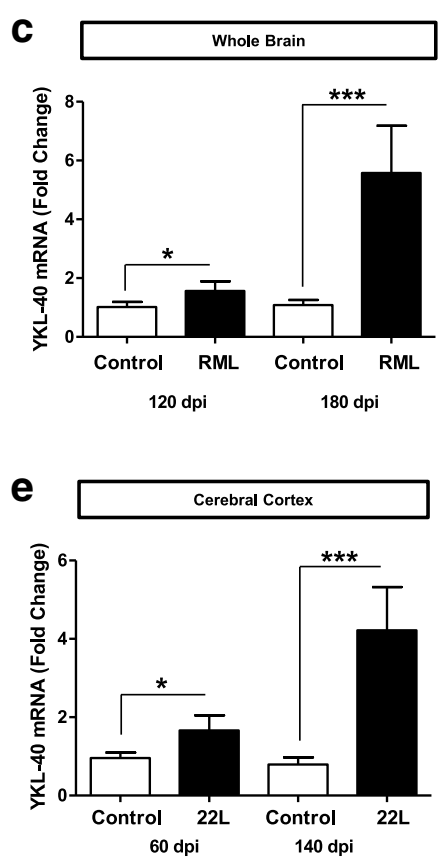

d
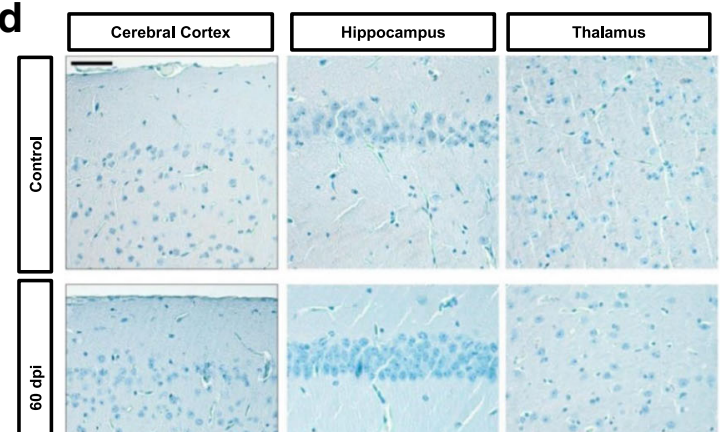

b
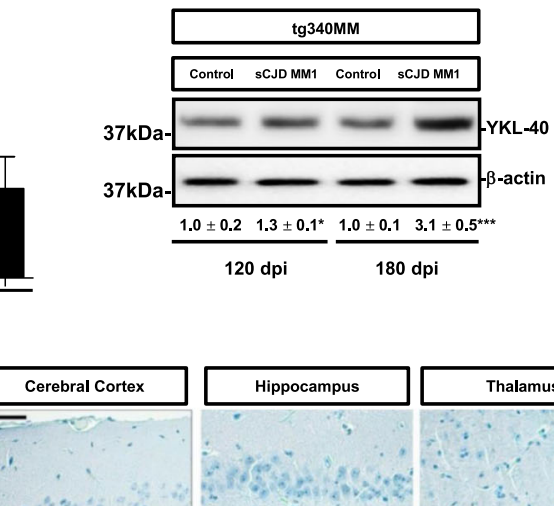

$\overline{\mathrm{a}}$

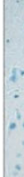

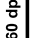

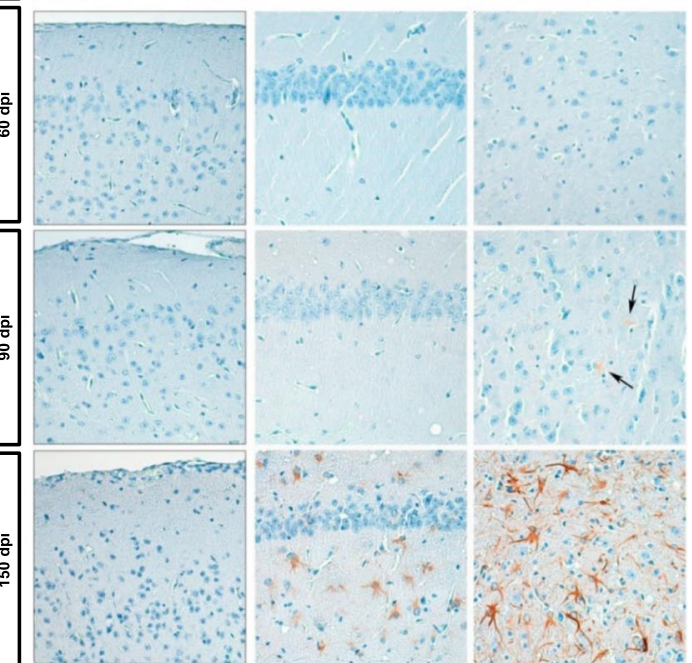

f

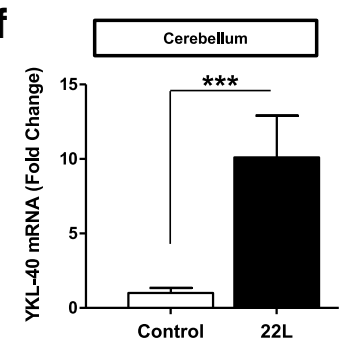

g
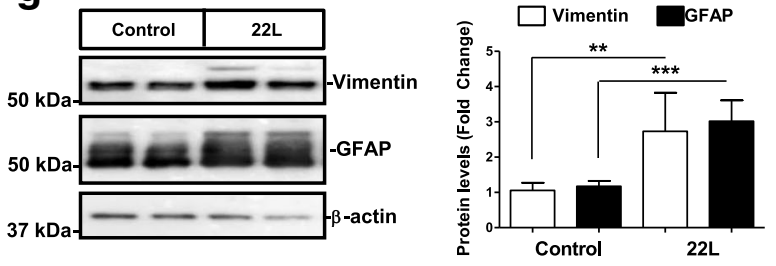

h
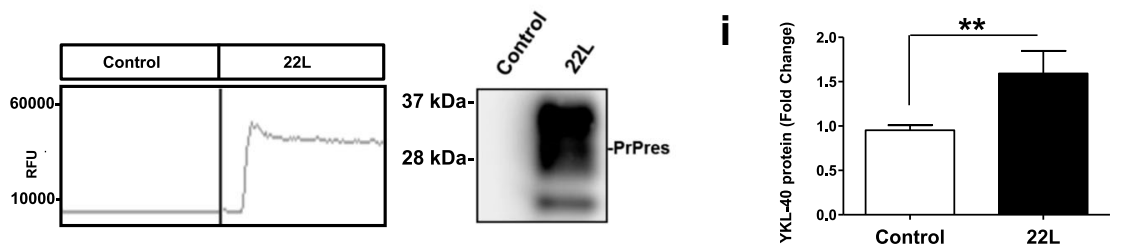

Fig. 3 (See legend on next page.) 


\begin{abstract}
(See figure on previous page.)
Fig. 3 YKL-40 expression in experimental models of prion diseases. a RT-qPCR analysis of YKL-40 in the cortex of control and sCJD MM1 inoculated tg340PRNP129MM mice at $120 \mathrm{dpi}$ (pre-clinical), $160 \mathrm{dpi}$ (early clinical), $180 \mathrm{dpi}$ (clinical) and $210 \mathrm{dpi}$ (clinical with 10-1 diluted inoculum). Four animals per group were analyzed. Normalization was performed using Hprt. b Representative Western-blot analyses for YKL-40 immunodetection in the cortex of control and SCID MM1-inoculated tg340PRNP129MM mice at 120 dpi (pre-clinical) and $180 \mathrm{dpi}$ (clinical). Three animals per group were analyzed. Normalization was based on $\beta$-actin levels. Numbers indicate densitometry results from three animals per group. Unpaired t-tests were performed to determine statistical differences. c RT-qPCR analysis of YKL-40 in the whole brain of control and RML scrapie-infected mice at pre-clinical (120 dpi) and clinical disease (180 dpi) stages. GAPDH was used for normalization. Similar results were acquired when normalization was based on Hprt expression levels (not shown). Unpaired t-tests were used for estimation of statistical differences. $\mathbf{d}$ Immunohistochemical analysis of YKL-40 expression in the cerebral cortex, hippocampus and thalamus of control and RML scrapie-infected mice at pre-clinical (60 and $90 \mathrm{dpi})$ and clinical (150 dpi) disease stages. Scale bar $=50 \mu \mathrm{m}$. Arrows indicate YKL-40 positive reactive astrocytes. Three animals per time point were used. Two sections were stained per animal (sagittal and coronal sections were used). Brown staining corresponds to YKL-40 staining and light blue to haematoxylin counterstaining. e RT-qPCR analysis of YKL-40 in the cerebral cortex of control and $22 \mathrm{~L}$ scrapie-infected mice at pre-clinical (60 dpi) and clinical (140 dpi) disease stages. $\mathbf{f}$ RT-qPCR analysis of YKL-40 in the cerebellum of control and $22 \mathrm{~L}$ scrapie-infected mice at clinical (140 dpi) disease stages. In all cases GAPDH was used for normalization. Similar results were obtained when Hprt was used for normalization (not shown). Unpaired t-tests were used to determine statistical differences. $\mathbf{g}$ Western blot analysis of Vimentin and GFAP in control and 22 L-scrapie infected COCS. $\beta$-actin was used for normalization. Graphic representation of densitometry analysis of five cerebellar tissues per condition is shown. Statistical differences were determined with unpaired t-tests. $\mathbf{h}$ RT-QulC analysis of control and $22 \mathrm{~L}$-scrapie infected COCS. A representative graph of three cerebellar tissues per condition is shown. All control tissues were tested negative, while all $22 \mathrm{~L}$-infected tissues were tested positive (left panel); western-blot showing the presence of PrPres in $22 \mathrm{~L}$-infected cells (right panel). i ELISA analysis of YKL-40 levels in control and $22 \mathrm{~L}$-scrapie infected COCS. Statistical differences were determined with unpaired t-tests. Fold changes in the expression of mRNA and protein were determined relative to the control cases. ${ }^{*} p<0.05,{ }^{* *} p<0.01,{ }^{* * *} p<0.001$
\end{abstract}

cells/mm2 and $3 \pm 2$ cells/mm2, respectively $(p<0.001)$ (Additional file 5A and B).

\section{YKL-40 expression in in vivo experimental models of prion disease}

To gain insight into the expression levels of YKL-40 in pre-clinical and early clinical sCJD stages, we took advantage of the tg340-PRNP-129MM mouse line inoculated with SCJD MM1 brain homogenate, which fully recapitulates the neuroinflammatory profile of human patients [40]. Analysis of the mRNA levels indicated that YKL-40 was increased at pre-clinical (120 dpi) and early-clinical (160 dpi) disease stages $(p<0.05)$. Differences between control and sCJD MM1-inoculated animals were more significant at clinical stages (180 dpi, $p<0.001$ and $210 \mathrm{dpi}$, $p<0.01$ ), resembling observations in $\mathrm{sCJD}$ postmortem brains (Fig. 3a). Western blots from the same set of inoculated animals validated the presence of elevated YKL-40 at pre-clinical $(p<0.05)$ and clinical $(p<0.001)$ disease stages at the protein level as well (Fig. 3b).

To ascertain whether increased YKL-40 expression was also present in non-human prion diseases, wild-type mice inoculated with $22 \mathrm{~L}$ and RML scrapie strains were utilized. Increased YKL-40 mRNA levels were detected in the whole brain of RML-infected animals at late preclinical (120 dpi, $p<0.05)$ and clinical (180 dpi, $p<0.001$ ) stages of the disease (Fig. 3c). Immunohistochemical analysis of RML-infected mice revealed the presence of YKL-40 positive astrocytes in the hippocampus and thalamus at clinical stages. In contrast to SCJD infected mice no staining was detected in cortical regions (Fig. 3d). At pre-clinical stages sparse positive
YKL-40 positive cells were detected in the thalamic nuclei (Fig. 3d). In contrast, mice inoculated with the $22 \mathrm{~L}$ strain, which led to a faster clinical onset and a distinct neuropathology including more cortical PrPSc deposition pattern, presented increased YKL-40 mRNA levels in the cerebral cortex at pre-clinical $(p<0.05)$ and clinical $(p<0.001)$ disease stages (Fig. 3e). Additionally, YKL-40 mRNA levels were highly increased in the cerebellum of $22 \mathrm{~L}$ infected mice (Fig. 3f) $(p<0.001)$.

\section{YKL-40 expression in ex vivo experimental models of prion disease}

The observation of increased YKL-40 in the cerebellum of $22 \mathrm{~L}$ infected mice allowed to speculate that $(22 \mathrm{~L})$-infected cultured organotypic cerebellar slices (COCS), which recapitulate most of the morphological and neuropathological hallmarks associated with prion infection and serve as an excellent ex vivo tool for the study of prion pathogenesis $[55,62]$, could recapitulate the alterations in YKL-40 expression observed in in vivo scrapie models. Ten weeks post-22 L infection, we detected astrogliosis by means of vimentin and GFAP expression (Fig. 3g). This was accompanied by positive seeding ability of pathogenic PrP by means of RT-QuIC analysis and presence of PrPres demonstrating the presence of prion infection on the COCS (Fig. 3h). At this time point, increased YKL-40 protein was detected in scrapie-infected COCS compared to mock treated cells $(p<0.01)$ (Fig. 3i).

\section{YKL-40 expression in AD patients and in AD experimental model}

Compared to controls, YKL-40 mRNA levels were increased in the FC of AD cases at early (AD I-III, 

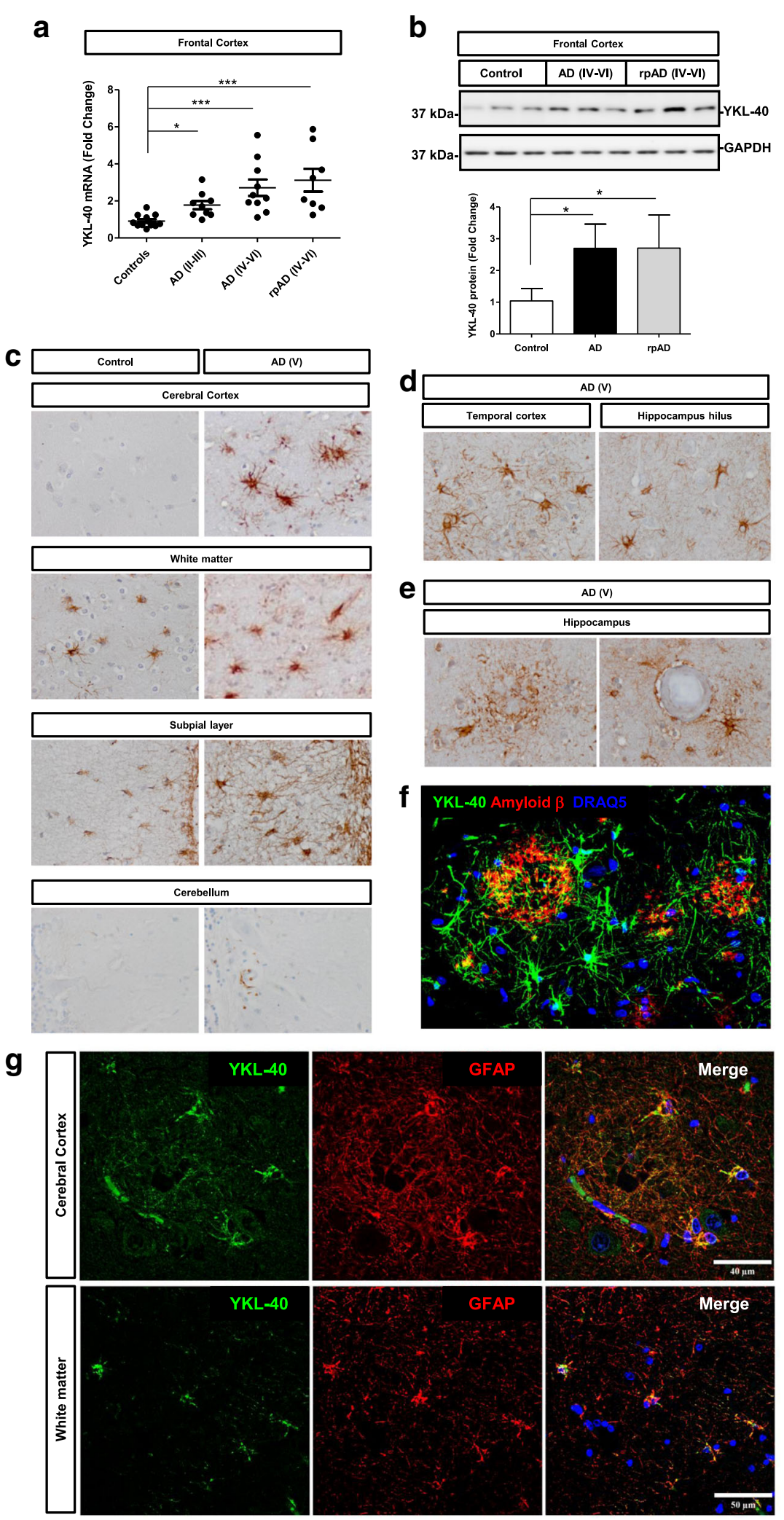

Fig. 4 (See legend on next page.) 


\begin{abstract}
(See figure on previous page.)
Fig. 4 YKL-40 expression in AD brain tissue (a) RT-gPCR analysis of YKL-40 in the frontal cortex of control, AD (I-III), AD (IV-VI) and rpAD (IV-VI) samples. GAPDH was used for normalization. Kruskal-Wallis and Dunn's post-hoc tests were used to estimate statistical differences. $\mathbf{b}$ Western blot analysis of YKL-40 in the frontal cortex of control, AD (IV-VI) and rpAD (IV-VI) samples. Normalization was based on GAPDH levels. Graphic summary of densitometry analyses performed on western blot results acquired from 8 control, 8 AD and 6 rpAD samples. c Immunohistochemical analysis of YKL-40 in the cerebral cortex, white matter, subpial layer and cerebellum in control and AD cases. $\mathbf{d}$ Immunohistochemical analysis of YKL-40 in the temporal cortex and hippocampus in AD cases. e Immunohistochemical analysis of YKL-40+ astrocytes surrounding $\beta$-amyloid plaques (left) and in blood vessels with amyloid angiopathy (right) in the hippocampal region of AD cases Brown staining corresponds to YKL-40 staining and light blue to haematoxylin counterstaining. $\mathbf{f}$ Double-labeling immunofluorescence of YKL-40 (green) and amyloid $\beta$ (red) in the hippocampus of AD. $\mathbf{g}$ Double-labeling immunofluorescence of YKL-40 (green) and GFAP (red) in cerebral cortex and white matter in AD tissues. Fold changes in expression of mRNA and protein were determined relative to the control cases. ${ }^{*} p<0.05$, ${ }^{* *} p<0.001$
\end{abstract}

$p<0.05)$ and late (AD IV-VI and rpAD IV-VI, $p<0.001$ ) neurofibrillary tangle Braak stages (Fig. 4a). Mean fold change values for controls versus AD comparisons were 1.8 for $\mathrm{AD}$ II-III and, 2.9 for AD IV-VI and 3.2 for rpAD IV-VI. No differences were detected between $\mathrm{AD}$ and rpAD cases $(p<0.05)$. YKL-40 mRNA levels were normalized with GAPDH, and similar results were obtained for GUSB normalization (Additional file 3B). In agreement with mRNA data, YKL-40 expression at protein level was increased in the FC of AD IV-VI (fold change 2.58) and rpAD IV-VI (fold change 2.59) cases $(p<0.05)$ (Fig. 4b).

Moreover, YKL-40 expression was analysed by immunohistochemical staining in several AD brain regions. Increased YKL-40 expression was detected in the cerebral cortex, white matter and subpial layer of $\mathrm{AD}$ sections compared to controls, while it remained unchanged in the cerebellum of $\mathrm{AD}$ cases (Fig. 4c). Increased expression was also detected in the temporal cortex and throughout the hippocampus region of $\mathrm{AD}$ brain sections (Fig. 4d, and Additional file 6A). YKL-40 staining was mainly detected in astrocytes, similarly to the patterns observed in SCJD cases. In contrast with the diffuse distribution of YKL-40-immunoreactive astrocytes in SCJD, YKL-40-positive astrocytes in the cerebral cortex in $\mathrm{AD}$ predominated in clusters often surrounding a core of fibrillar $\beta$-amyloid (Fig. 4e). YKL-40positive astrocytes were also present around blood vessels with $\beta$-amyloid (Fig. 4e and Additional file 6B). Double-labelling with YKL-40 and $\beta$-amyloid antibodies was used to demonstrate the presence of YKL-40 positive astrocytes surrounding senile and diffuse $\beta$-amyloid plaques in the hippocampus of $\mathrm{AD}$ cases (Fig. $4 \mathrm{f}$ and Additional file 7).

Fibrillar astrocytes in the subcortical white matter and deep white matter were also strongly YKL-40 immunoreactive. Specific expression of YKL-40 in GFAP-immunoreactive astrocytes in the cerebral cortex and white matter regions (Fig. 4g) in $\mathrm{AD}$ brains was confirmed by double-labelling immunofluorescence and confocal microscopy. Quantitative assessment of double-labelled GFAP and YKL-40 cells indicated a significant superior number of astrocytes showing co-localization of GFAP and YKL-40 (238 \pm 23 cells $/ \mathrm{mm} 2)$, compared to those showing only GFAP or only YKL-40 staining (28 \pm 6 cells $/ \mathrm{mm} 2$ and $17 \pm 3$ cells $/ \mathrm{mm} 2$, respectively) $(p<0.001)$ (Additional file 5C and D).

As an in vivo model of $A D$ pathogenesis we used the $5 x F A D$ mouse model. At 3 months of age, 5xFAD animals presented glial changes and amyloid plaques but do not show clinical signs or behavioural alterations [53, 54]. At this time point, we found increased YKL-40 and GFAP protein levels $(p<0.05)$ (Additional file 8A). At immunohistochemical level, widespread astrocytic YKL40 expression was detected in 10-month-old 5xFAD animals, especially in the cerebral cortex and hippocampus (Additional file 8B) as well as in the thalamus, caudate putamen and midbrain, while sparse YKL- 40 positive astrocytes could be detected in the molecular layer of cerebellum. At this stage, animals showed clear clinical and behavioural signs.

\section{YKL-40 in DLB}

YKL-40 mRNA expression was further analysed in the FC of DLB cases. Despite increased mean values of YKL.40 detected in DLB and rapid forms of DLB (rpDLB) compared to controls, no statistically significant alterations were found between these groups (Fig. 5a). Similarly, no differences at the protein level were detected between control and DLB cases (Fig. 5b). Sparse YKL-40-positive astrocytes were observed in the cortical regions, white matter and subpial layer in DLB cases; sparse clusters of YKL-40-positive astrocytes were not uncommon due to the fact of the associated low burden of AD-related pathology in DLB (Fig. 5c).

\section{CSF YKL-40 in the differential diagnosis of neurodegenerative dementias}

When CSF YKL-40 was analysed in the differential diagnostic context of neurodegenerative dementias (cohort 1 , Additional file 2), significantly higher YKL-40 levels were detected in SCJD cases $(480 \pm 178 \mathrm{pg} / \mathrm{mL})$ compared to neurological controls (NC) $(250 \pm 77 \mathrm{pg} / \mathrm{mL})$ and to the other dementia groups $(p<0.001)$ (Fig. 6a, Additional file 2). Compared to NC, YKL-40 was also increased in $\mathrm{AD}$ patients $(386 \pm 221 \mathrm{pg} / \mathrm{mL}, p<0.001)$ but not in $\mathrm{VaD}(323 \pm 96 \mathrm{pg} / \mathrm{mL}, p>0.05)$ and $\mathrm{DLB} /$ 

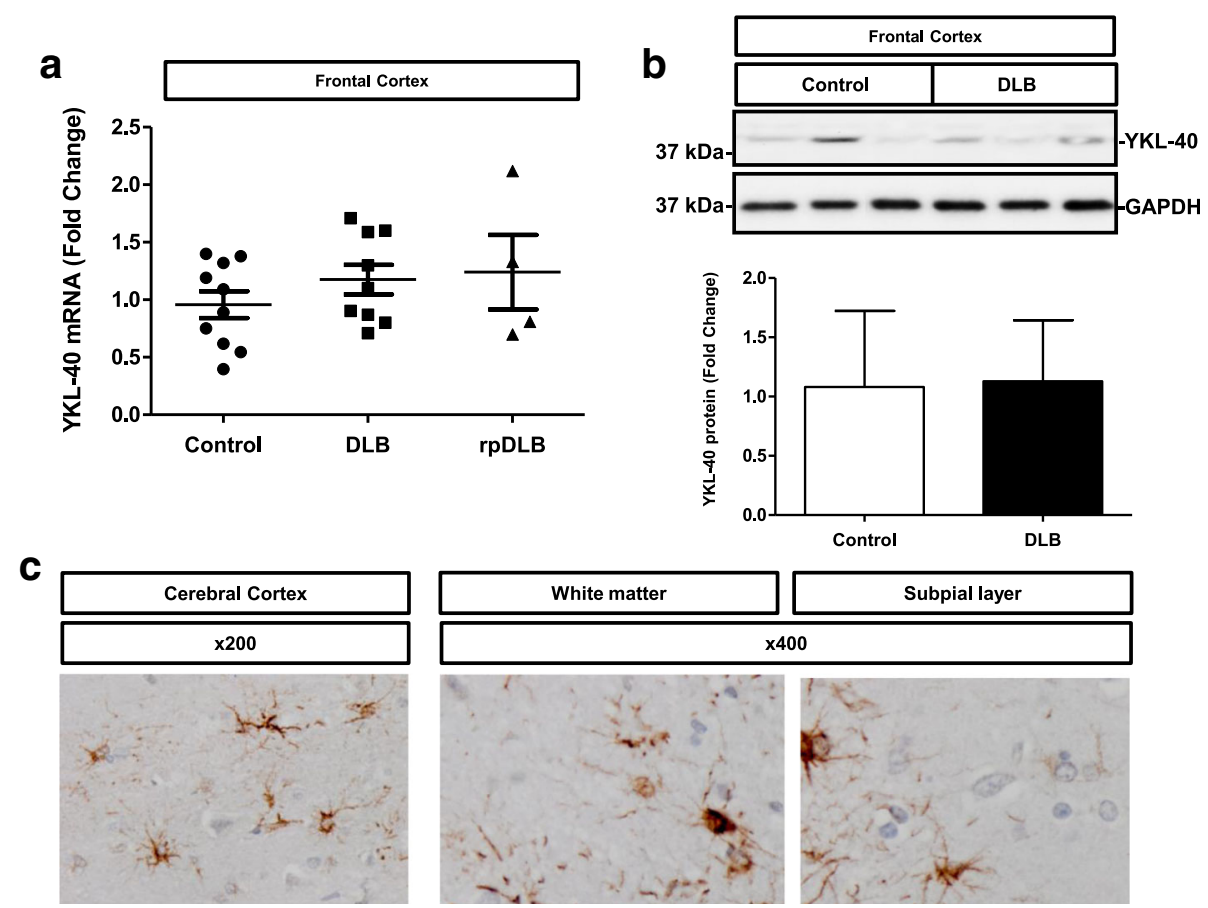

Fig. 5 YKL-40 expression in DLB brain tissue. a RT-qPCR analysis of YKL-40 in the frontal cortex of control, DLB and rpDLB samples. Normalization was based on GAPDH levels. $\mathbf{b}$ Western blot analysis of YKL-40 in the frontal cortex of control, DLB and rpDLB samples. Normalization was carried out with GAPDH. Kruskal-Wallis and Dunn's post-hoc tests were used for determination of statistical differences. c Immunohistochemical analysis of YKL-40 in the cerebral cortex, white matter and subpial layer in DLB cases. Brown staining corresponds to YKL-40 staining and light blue to haematoxylin counterstaining. Fold changes in expression (mRNA and protein) were determined relative to the control cases

PDD cases $(314 \pm 103 \mathrm{pg} / \mathrm{mL}, p>0.05)$. To calculate the clinical accuracy of YKL-40 in discriminating between dementia and NC groups, we estimated the AUC values. As expected, the highest values were detected when comparing $\mathrm{NC}$ and sCJD cases (AUC: 0.92, 95\% CI: 0.87-0.96) (Fig. 6b). Using an optimal cut-off at $315 \mathrm{pg} /$ $\mathrm{mL}$ defined by the Youden index, an overall sensitivity of $85 \%$ and specificity of $84 \%$ could be achieved. For the rest of the dementias AUC values were far below those detected for sCJD cases: NC vs AD; AUC: $0.77,95 \% \mathrm{CI}$ : 0.68-0.84, NC vs VaD; AUC: 0.71, 95\% CI: 0.60-0.84 and NC vs DLB: AUC: 0.70, 95\% CI: 0.60-0.80 (Fig. 6b). Next we explored the ability of CSF YKL-40 to discriminate sCJD from the rest of dementia groups. While YKL-40 could not discriminate SCJD from AD with high accuracy (AUC: 0.70, 95\% CI: 0.62-0.78), values were higher for sCJD vs DLB/PDD (AUC: 0.81, 95\% CI: $0.72-0.89$ ) and SCJD vs VaD (AUC: $0.77,95 \%$ CI: 0.67-0.88) comparisons.

Further we analysed whether YKL-40 levels could be influenced by the rate of cognitive decline and/or disease duration. For this reason, we stratified AD cases into slow and fast progressive $\mathrm{AD}(\mathrm{AD}$ versus $\mathrm{rpAD})$ and $\mathrm{SCJD}$ samples according to codon 129PRNP polymorphism, which plays a role in the clinico-pathological phenotype of the disease and, as a consequence, in disease duration $[43,63$,
64]. No differences in CSF YKL-40 levels were observed between $\mathrm{AD}(387 \pm 244 \mathrm{pg} / \mathrm{mL})$ and $\mathrm{rpAD}(384 \pm 128 \mathrm{pg} /$ $\mathrm{mL}$ ) cases or between sCJD cases according to their codon 129 polymorphism: sCJD MM $(462 \pm 195 \mathrm{pg} / \mathrm{mL}), \mathrm{MV}$ $(519 \pm 203 \mathrm{pg} / \mathrm{mL})$ and VV $(456 \pm 79 \mathrm{pg} / \mathrm{mL})$ (Fig. 6c, d).

Next we sought to investigate the potential correlation between YKL-40 and tau, disease duration and age in SCJD cases. A significant positive correlation of YKL-40 with CSF tau was found $(p<0.05)$ (Fig. 7a), but no correlation of YKL-40 levels with CSF A $\beta 42$, disease duration and age at disease onset was detected $(p>0.05)$ (Fig. 7b and c).

\section{Validation of increased YKL-40 levels in the CSF of prion diseases}

In order to validate the presence of increased YKL-40 levels in SCJD, two independent cohorts were used. The first validation cohort (cohort 2, Additional file 2) included healthy controls (HC), AD and SCJD cases. Compared to HC (226 $\pm 82 \mathrm{pg} / \mathrm{mL})$, YKL-40 levels were significantly increased in SCJD $(411 \pm 157 \mathrm{pg} / \mathrm{mL}$, $p<0.001)$ and $\mathrm{AD}(311 \pm 113 \mathrm{pg} / \mathrm{mL}, p<0.01)$ (Fig. $8 \mathrm{a}$, cohort 2 Additional file 2). Similar results were obtained for the second validation cohort (cohort 3, Additional file 2), where YKL-40 levels in SCJD were significantly higher $(603 \pm 198 \mathrm{pg} / \mathrm{mL})$ than those detected in cognitive impairment/dementia cases $(358 \pm 129 \mathrm{pg} / \mathrm{mL}$, 


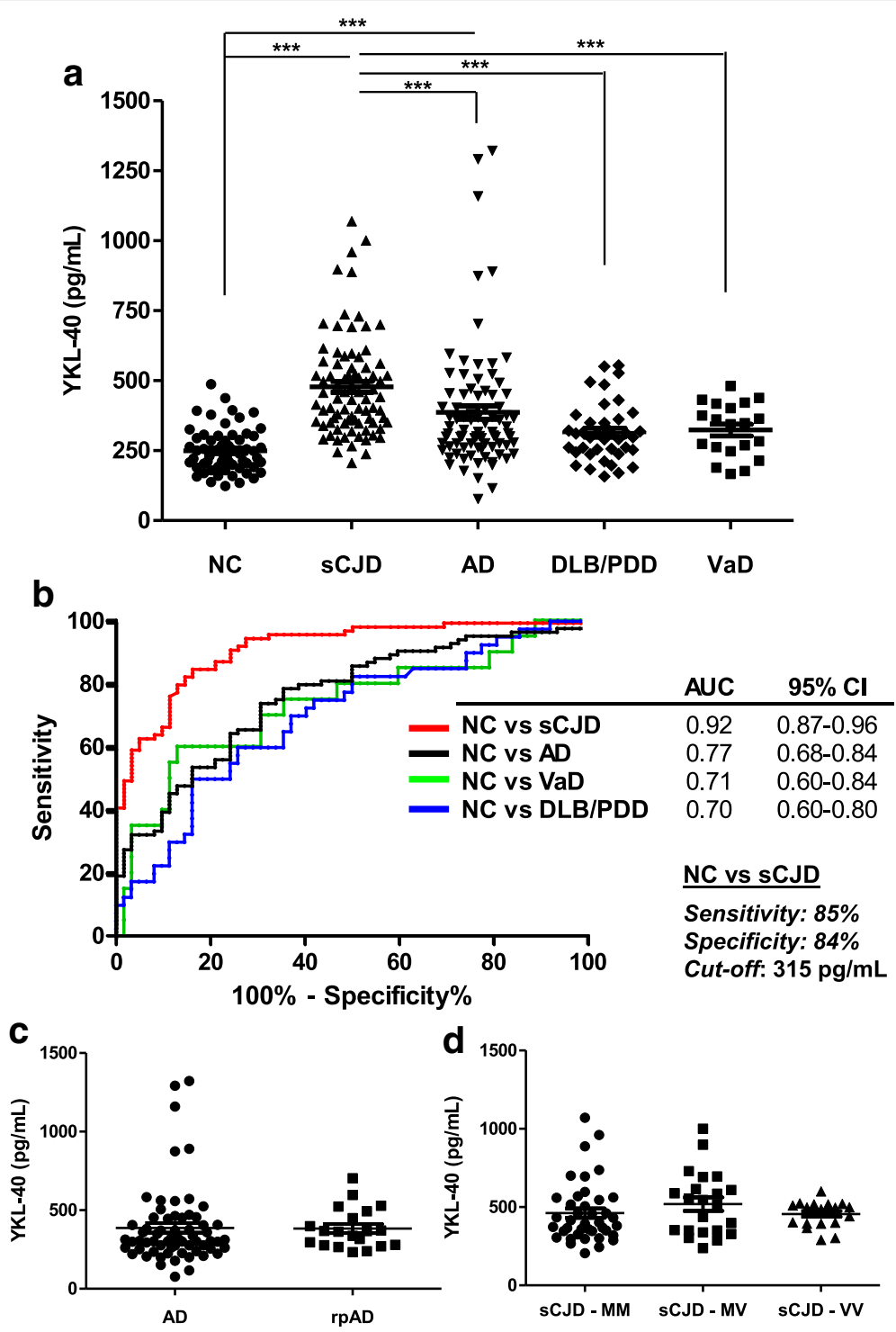

Fig. 6 CSF YKL-40 in the differential diagnosis of neurodegenerative dementia. a CSF YKL-40 in NC $(n=62), \mathrm{sCJD}(n=84), \mathrm{AD}(n=84)$, DLB/PDD $(n=40)$ and $\operatorname{VaD}(n=20)$ cases (cohort 1). Number of cases analyzed and statistical significance of differences between groups is indicated. Kruskal-Wallis and Dunn's post-hoc tests were used for estimation of statistical differences. $\mathbf{b}$ ROC curves for YKL-40 quantification in the differential diagnosis of dementia groups compared to NC. In the legend, AUC values, corresponding to the area under ROC curves, and 95\% confidence intervals are reported. Diagnostic parameters (cut-off, sensitivity and specificity) are indicated for NC versus SCJD comparison. c Stratification of AD cases in slow progressive (AD) $(n=64)$ and rapid progressive $(\mathrm{rpAD})(n=20)$ cases. Statistical differences were tested with Mann-Whitney test. $\mathbf{d}$ Stratification of sCJD cases according to PRNP codon 129 polymorphism (MM, $n=40, M V, n=22$ and W, $n=20$ ). Kruskal-Wallis and Dunn's post-hoc tests were used for statistical differences estimation. ${ }^{* * *} p<0.001$

$p<0.01$ ) (Fig. 8b, cohort 3 Additional file 2). Finally, we aimed to determine the usefulness of CSF YKL-40 levels in the discrimination of prion disease from genetic etiology. For this purpose, we analysed genetic cases from two cohorts and compared them with their respective controls and sCJD groups. The study included Controls, sCJD, gCJD PRNP-E200K and Fatal Familial Insomnia (FFI) (PRNP-D178N mutation) cases (cohort 1 and cohort 2, Additional file 2). In both cohorts gCJD cases harbouring the E200K mutation showed similar YKL-40 levels compared to sCJD cases and were statistically different from controls $(p<0.001)$. In contrast, YKL40 levels in FFI cases were lower than in SCJD cases, but significantly higher compared to control subjects $(p<0.05)$ (Fig. 9a and $\mathrm{b}$ and cohort 1 and cohort 2, Additional file 2).

\section{Discussion}

Neuroinflammation in dementia has been identified as a major contributor to disease pathogenesis and as a 


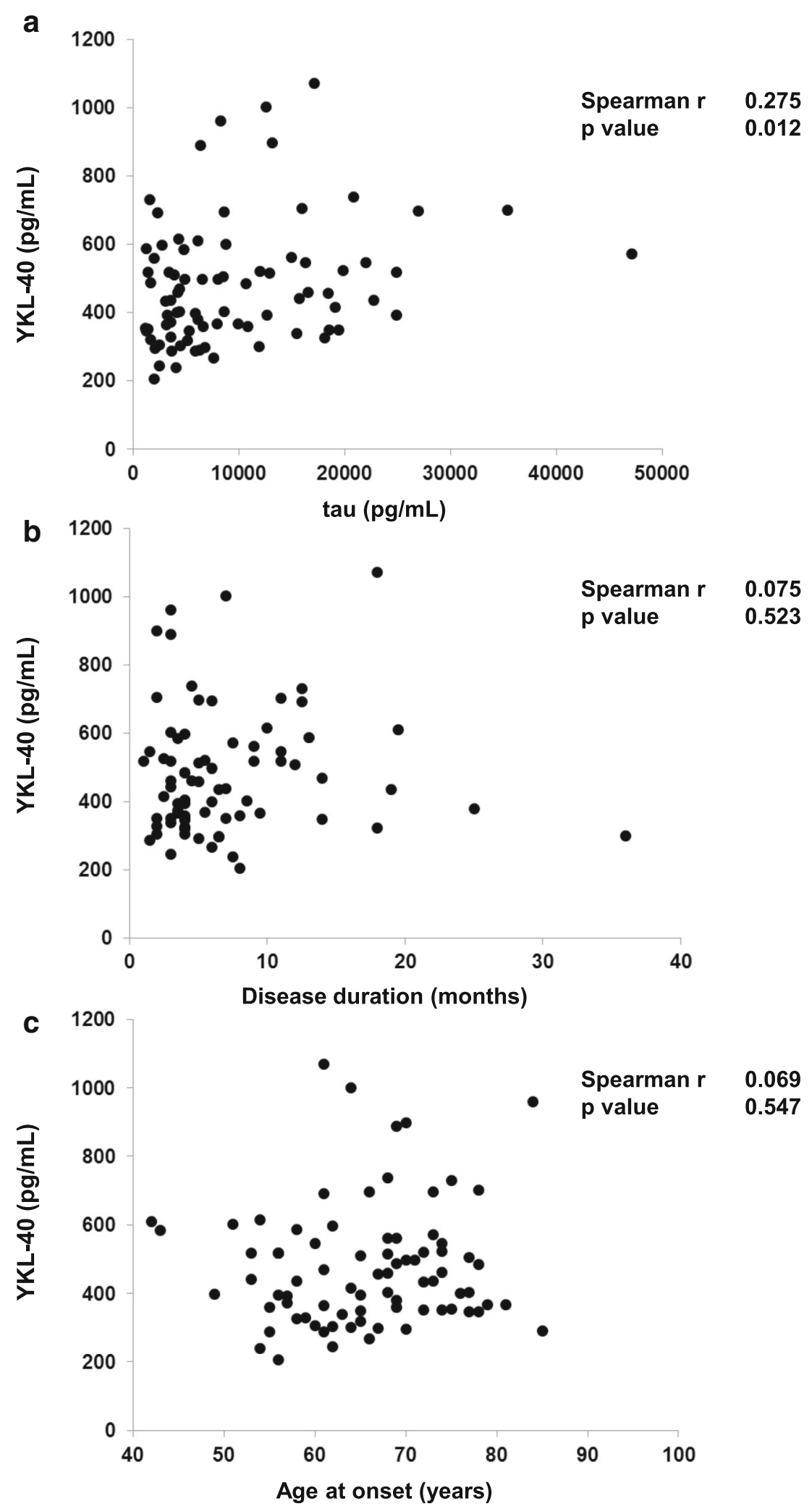

Fig. 7 (See legend on next page.) 
(See figure on previous page.)

Fig. 7 Correlation of CSF YKL-40 levels with CSF tau levels, disease duration and age at onset. Correlation analysis between CSF YKL-40 and (a) CSF tau levels, (b) disease duration (as time in months from disease onset to death) and (c) age at onset. The association between CSF YKL-40 and either biomarkers or demographics parameters was investigated with the Spearman correlation. Positive correlation was detected only for CSF YKL-40 versus CSF tau comparison

potentially causative effect of neurodegeneration in chronic profiles [65-67]. However, the precise regional and temporal contribution of glial activation to the pathogenic process is far from understood. Similar to microglial activation, sustained astrocytic activation may lead to a switch from a neuroprotective and neurotrophic to a neurodegenerative phenotype, increasing neuronal vulnerability to chemokines, pro-inflammatory cytokines and reactive oxygen species [68-70]. This is exemplified by proteins such as $\mathrm{S100B}$, which is released from astrocytes with neurotrophic or neurotoxic effects on neurons depending on their concentration and on the astrocytic activation state [71, 72]. In turn, increased expression and secretion of S100B from astrocytes may

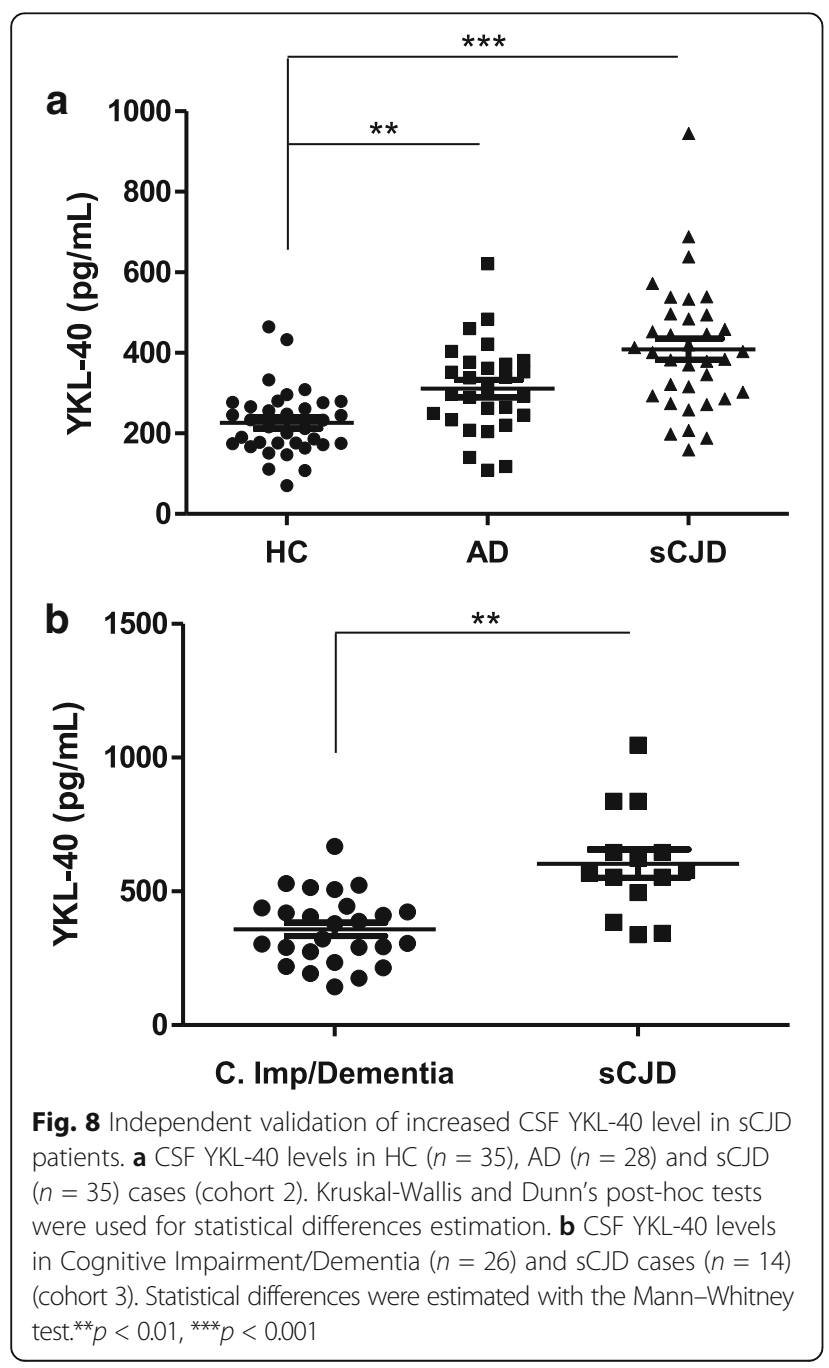

be reflected in the CSF patterns as reported in traumatic brain injury [73], $\mathrm{AD}[74,75]$ and sCJD [76]. Consequently, astrocytes are turning into a potential target for therapeutic intervention in neurodegenerative diseases associated with an inflammatory component [77], indicating that astrocyte-associated metabolites may be potential biomarkers in biological fluids. Thus, studies on expression patterns and function of secreted, astrocytic-

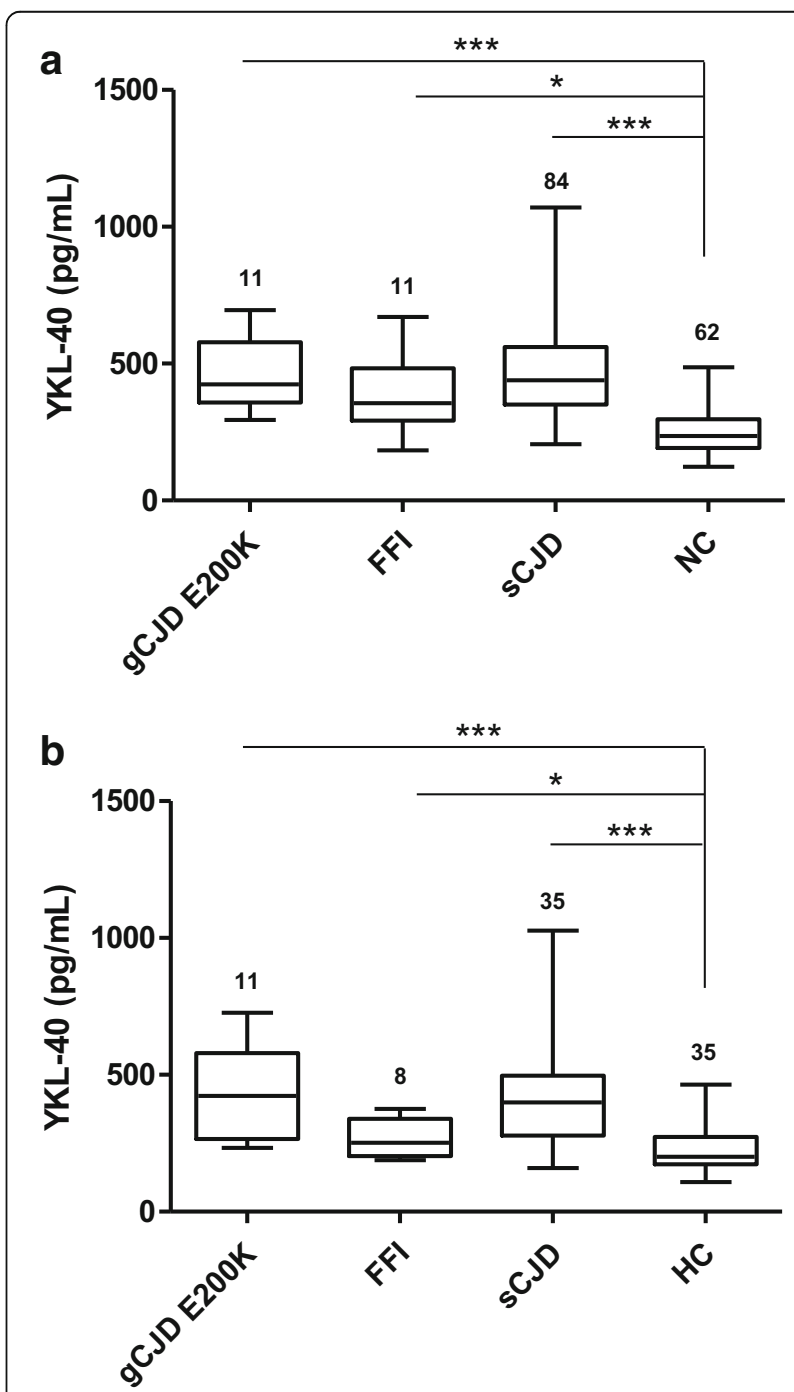

Fig. 9 Detection of CSF YKL-40 levels in gCJD E200K and Fatal Familial Insomnia. a CSF YKL-40 levels in gCJD E200K, Fatal Familial Insomnia (FFI) and NC (cohort 1). b CSF YKL-40 levels in gCJD E200K, Fatal Familial Insomnia (FFI) and HC (cohort 2). Statistical differences were determined with Kruskal-Wallis and Dunn's post-hoc tests. ${ }^{*} p<0.05,{ }^{* *} p<0.001$ 
related molecules under pathological or stress conditions are gaining experimental momentum.

The functional role of YKL-40 during neuroinflammation is unclear. However, a neuroprotective effect has been assigned to YKL-40 by an in vivo study, according to which YKL-40 knock-out mice presented more severe neuropathology and more prominent gliosis than their wild-type littermates in a model of controlled cortical impact [78]. These results indicated that YKL-40 modulates the neuroinflammatory response associated with traumatic brain impact (TBI). In agreement with this, YKL-40 knock-out mice present exacerbated experimental autoimmune encephalomyelitis clinical scores accompanied by increased lymphocytic and macrophage infiltrates and gliosis compared to wild-type animals [79], suggesting that YKL-40 is necessary for the proper resolution of inflammation. Importantly, astrocytic YKL-40 expression is under the control of microglial stimuli since YKL-40 transcription, accompanied by morphological changes and increased migratory capacity characteristic of reactive gliosis, is induced by the macrophage-released proinflammatory mediators Interleukin-1 $\beta$ (IL-1 $\beta$ ) and tumor necrosis factor- $\alpha$ [22]. Interestingly, both cytokines are highly expressed in the microglia of TBI, sCJD, AD and related mouse models $[40,41,80]$, suggesting a cross-talk between microglial and astrocytic activation in the regulation of YKL-40-mediated functions in chronic inflammatory profiles associated with neurodegeneration. These data are supported by the finding that YKL-40 expression correlates with the expression of IL-1 $\beta$ and Interleukin- 6 and that both cytokines are able to synergistically up-regulate YKL-40 expression in primary astrocytes in vitro in a STAT3 dependent-manner [81], which in turn, has been seen to be activated in the FC of sCJD brains [40]. In this regard, it has been recently shown that a subtype of reactive astrocytes present in neurodegenerative diseases is induced by secreted cytokines from activated microglia [82], reinforcing the suggested role of astrocyte-microglia cross-talk during neurodegeneration [83, 84].

Aiming at a better characterization of YKL-40's role in neurodegenerative disorders, we studied its mRNA and protein levels and its expression in different brain regions in several neurodegenerative conditions. Astrocytic YKL-40 overexpression was prominent in both SCJD and AD, in agreement with the wellknown chronic inflammatory profile of both diseases [38]. Most of the YKL-40+ astrocytes were stained with GFAP antibodies, both in SCJD and AD. However, a small population of astrocytes with single labelling for one of the two markers was also detected, in agreement with the positive but moderate correlation between GFAP and YKL-40 expression levels detected in sCJD.
Further, the absence of YKL-40 overexpression in DLB was in line with the corresponding low inflammatory profile in DLB $[42,85]$. Disease-specific differences were not restricted to expression levels, but also included regional-dependent regulation. Indeed, we demonstrated that YKL-40 overexpression in the cerebellum is restricted to SCJD and absent in AD.

Data obtained from prion models suggest a role of YKL-40 at pre-clinical and early stages of the disease. In RML infected mice, early prion pathology including microglia activation, astrogliosis and PrPSc deposition could be first detected in the thalamus, whereas hippocampus and cortex still remain unchanged at the microscopic level. At clinical disease stages, hippocampus and cerebellum show widespread signs of prion pathology. This is in line with our detection of YKL-40 immunoreactivity in RML infected mice, which was abundant in the thalamus only at preclinical time points, but was more widespread, including hippocampus, at clinical prion disease stages. This is supported by the elevated levels of YKL-40 in cortical region of the sCJD model at pre-clinical disease stage.

Incorporation of new CSF biomarkers in clinical practice not only improves current diagnostic approaches across the disease continuum, but also provides new tools for prognosis and evaluation of therapeutic efficacy once disease-modifying treatments become available [86-88]. Therefore, peripheral biomarkers mirroring specific disease hallmarks such as neuronal/synaptic damage, neuroinflammation and the presence of misfolded proteins will become useful to evaluate therapeutic approaches. In this regard, we demonstrated that CSF YKL-40 patterns resemble expression levels in brain tissue in a disease-specific manner. Additionally, we detected elevated YKL-40 in the brain of early AD (Braak stages I-III). Although data regarding elevated CSF YKL40 in early stages of AD are contradictory [24, 25, 89, 90], our results support the presence of increased astrocytic YKL-40 expression, together with a role of astrocytosis, in early AD pathogenesis [91]. Similarly, we detected increased YKL-40 in the brain of an AD mouse model and in experimental models of prion diseases at pre-clinical stages, supporting the presence of astrocytic alterations before clinical onset and the usefulness of YKL-40 quantification in the detection of early pathological changes associated with neuroinflammation. Interestingly increase in YKL-40 at pre-clinical stages (120 dpi) of the sCJD mice model occurs when PrPres, but not synaptic damage, is already detectable [92] [Llorens et al. 2017, submitted], supporting the hypothesis that neuroinflammation may precede neuronal dysfunction.

Additionally, it has recently been shown that in $\mathrm{MCI}$ and pre-AD stages, APOE $\varepsilon 4$ carriers display higher CSF levels of YKL-40 but not of a marker of microglial 
activation, sTREM2 (soluble triggering receptor expressed on myeloid cells 2), supporting a role of APOE $\varepsilon 4$ in the regulation of astroglial response in AD pathology [93]. However, CSF from pre-sCJD stages is not available to rule out a potential role of CSF YKL-40 as pre-clinical marker of prion diseases. In this regard, use of in vivo prion models not only validates regional alterations observed in human tissue, but also supports utilization of humanized SCJD mouse models in the understanding of human pathogenesis at pre-clinical disease stages, since scrapie infected animals present strainspecific regional alterations that differ from those detected in human and mouse SCJD samples.

An interesting finding of our study is the detection of increased CSF YKL-40 levels in FFI, supporting the idea that astrogliosis is a common hallmark in FFI brain tissue even in regions with no major pathological involvement [94, 95]. In contrast, CSF surrogate markers of neuronal damage are less sensitive in detecting FFI cases $[44,96]$ as neuronal damage is restricted to thalamic nuclei. Elevated YKL-40 levels in gCJD-E200K patients were in line with the similar diagnostic parameters between SCJD and gCJD-E200K for other prion biomarkers $[44,96]$, due to the common clinicopathological spectrum of gCJD-E200K and sCJD cases [97, 98]. Despite high CSF YKL-40 levels were found in sCJD and AD, increased YKL-40 levels have also been detected in other neurological and psychiatric conditions such as brain concussions [99], acute ischemic stroke [100], suicidal ideation [101] and bipolar disorder [102], underlining that astrocytic activation is not restricted to neurodegenerative conditions and that neuroinflammation may play a more prominent and common role in the etiology of neurological diseases.

Conflicting data have been reported on the correlation of YKL-40 with markers of neurodegeneration [23, 25, $31,32,89]$ as well as with cognitive decline [28, 31, 36, 89]. The distinct correlations between different surrogate disease markers and cognitive decline suggest that dementia hallmarks may reflect different aspects of neurodegeneration processes which, although potentially associated, may provide independent information about the pathological state of the brain tissue. In this regard, it has been proven that, in early AD, CSF YKL-40 is associated with a cerebral structural signature distinct from that related to p-tau neurodegeneration [28], even when a positive correlation between the levels of the two biomarkers in the CSF is reported [23-25, 34].

For sCJD, the positive correlation between CSF tau and YKL-40 suggests an association between axonal damage and neuroinflammation. Indeed, it is well known that inflammatory patterns in SCJD correlate with the severity of the neuropathological lesions [103]. In contrast, no correlation was detected between age and disease duration, most likely due to the very short disease duration of sCJD cases (mean disease duration $=7.0 \pm 5.9$ months).

\section{Conclusions}

Here we report, for the first time, that higher YKL-40 levels are detectable in the brain and CSF in prion diseases. Further we show that CSF YKL-40 presents a good diagnostic accuracy in the discrimination of $\mathrm{sCJD}$ from NC. While YKL-40 is also elevated in AD, it failed discriminating $\mathrm{AD}, \mathrm{VaD}$ and $\mathrm{DLB} / \mathrm{PDD}$ cases (AUC < 0.8 ). In this regard, caution should be taken when interpreting elevated CSF YKL-40 levels in the diagnostic context of neurodegenerative dementias. However, since CSF YKL-40 resembles astrocytic activation in brain tissue we speculate that YKL-40 quantification may be useful for the assessment of neuroinflammatory profiles during therapeutic intervention.

\section{Additional files}

Additional file 1: Summary of human brain cases and regions analyzed with qPCR, western blot and immunohistochemical analysis. Number of cases, age (mean $\pm \mathrm{SD}$ ) and gender (number of females and males) are indicated. (TIFF $93 \mathrm{~kb}$ )

Additional file 2: Summary of CSF samples from the three independent cohorts used in this study. Sample group, number of cases, age (mean $\pm \mathrm{SD}$ ), tau levels (pg/mL), A 442 levels (pg/mL), 14-3-3 positive cases and $Y K L-40$ levels $(\mathrm{pg} / \mathrm{mL})$ are indicated. (TIFF $147 \mathrm{~kb})$

Additional file 3: YKL-40 mRNA expression in SCJD and AD using GUSB as housekeeping gene. Validation of alterations in YKL-40 mRNA in SCJD (A) and AD (B) cases using GUSB as qPCR housekeeping gene. (TIFF $59 \mathrm{~kb}$ )

Additional file 4: YKL-40-positive astrocytes associated to PrP amyloid plaques. Immunofluorescence images obtained from double-labeling staining with GFAP (A) and YKL-40 (B) (green) and PrP (red) antibodies in the cortex of SCJD. Individual channels as well as merge images are shown for two different cortical areas. (TIFF $768 \mathrm{~kb}$ )

Additional file 5: Quantification of YKL-40 and GFAP overlap in SCJD and $A D$ cases. Immunofluorescence images obtained from double-labelling staining with GFAP (red) and YKL-40 (green) antibodies in the hippocampus of SCJD ( $n=3)(A)$ and AD V $(n=3)(C)$ cases. Individual channels as well as merge images are shown for two different cortical areas (one cortical image per CJD or AD patient) of two different patients. Quantifications of GFAP + YKL-40+ (YKL-40-GFAP), GFAP+/YKL-40- (GFAP) and GFAP-/YKL-40+ (YKL-40) astrocytes are shown. Statistical significance differences were detected between YKL-40-GFAP and YKL-40, GFAP groups; \#\#\#p $<0.001$ and ${ }^{* *} p<0.001$, respectively. (TIFF $5257 \mathrm{~kb}$ )

Additional file 6: $\mathrm{YKL}-40$ expression in $\mathrm{AD}$. (A) $\mathrm{YKL}-40$ expression in astrocytes in the dentate gyrus of AD. (B) Immunohistochemical analysis of YKL-40+ astrocytes surrounding $\beta$-amyloid plaques in AD cases. (TIFF 989 kb)

Additional file 7: $Y K L-40$ positive astrocytes associated to $\beta$-amyloid plaques. Confocal Z-stack images obtained from double-labelling staining with YKL-40 (green) and $\beta$-amyloid (red) antibodies in the hippocampus of $A D$ tissue. Z-stacks from representative senile $\beta$-amyloid plaque (A) and diffuse amyloid plaque (B) is shown. DRAQ5 staining is shown in blue. Individual channels as well as merge images are shown. Distance between- $Z$ Stacks sections $=0.5 \mu \mathrm{m}$. (ZIP $2287 \mathrm{~kb})$

Additional file 8: YKL-40 expression in 5xFAD mice. (A) Western blot analysis of YKL-40 and GFAP in the cortex of 3-month-old control and 5xFAD animals. Four animals per group were analyzed. GAPDH was used for normalization. Numbers represent the summary of densitometric analysis. Unpaired t-test was used for statistical differences estimation. (B) Immunofluorescence analysis of YKL-40 (green) in the cerebellar cortex and 
hippocampus of 10-month-old 5XFAD mice. Nuclei were stained with DAPI (blue). Three animals per group and three sections per animal were analyzed. (TIFF $235 \mathrm{~kb}$ )

\section{Acknowledgements}

The authors thank Kristin Hartmann (Core facility mouse pathology/UKE) and Dr. Thomas Meyer (Department of Psychosomatic Medicine/UMG) for assistance on animal experimentation and Silja Koechy and Nadine Gotzmann (UMG/DZNE) for technical assistance. We also thank Benjamín Torrejón Escribano (CCiT-UB) for the technical support. We are indebted to the Neurological Tissue Bank of the Biobanc-Hospital Clinic-IDIBAPS for sample procurement.

\section{Funding}

This study was funded by Robert Koch Institute through funds from the Federal Ministry of Health of Germany (grant no. 1369-341) to IZ, by CIBERNED (Network Center for Biomedical Research of Neurodegenerative Diseases) project BESAD-P to IF, by the Spanish Ministry of Health - Instituto Carlos III/ Fondo Social Europeo (CP16/00041) to FL, by the Red Nacional de Priones (AGL2015-71764-REDT- MINECO) to FL, IZ, JMT, RSV and IF and by the IKYDA project (ID 57260006) to FL, IZ and TS.

\section{Availability of data and materials}

The datasets used and/or analysed during the current study are available from the corresponding author on reasonable request.

\section{Author's contributions}

Study conception and design: FL, IF, IZ. Project supervision: FL. Data collection: $F L, K T, W T, K X$, EK, EKo, CP, DD-L, MS, DO, SC, AC, PG-E, IM, OA, NFB, IMV, MG, TS, JMT, SK, RSV, IF, IZ. Data analysis and interpretation: FL, KT, WT, MG, SK, IF, IZ. Drafting the article: FL, IF. Final approval of the version to be published: All authors.

\section{Ethics approval and consent to participate}

The present study was conducted according to the revised Declaration of Helsinki and Good Clinical Practice guidelines and was approved by the local ethics committees. Informed consent was given by all study participants or their legal representative. For animal investigation, principles of laboratory animal care (NIH publication No. 86-23, revised 1985) were followed. All animal experiments were performed in compliance with the French, German and Greek national guidelines, in accordance with the European Community Council Directive 86/609/EEC and approved by local ethics committees. The experimental protocols were approved by the INRA Toulouse/ENVT ethics committee, the Ethical Committee of the Freie und Hansestadt Hamburg, Amt für Gesundheit und Verbraucherschutz (Permit number: G 21307/591-00.33) and the AHEPA University Hospital local veterinary authorities (21/12/2015).

\section{Competing interests}

The authors declare that they have no competing interests.

\section{Publisher's Note}

Springer Nature remains neutral with regard to jurisdictional claims in published maps and institutional affiliations.

\section{Author details}

${ }^{1}$ Network Center for Biomedical Research in Neurodegenerative Diseases (CIBERNED), Institute Carlos III, Ministry of Health, Feixa Llarga s/n, 08907 L'Hospitalet de Llobregat, Barcelona, Spain. ${ }^{2}$ Department of Neurology, University Medical School, Göttingen, Germany. ${ }^{3}$ German Center for Neurodegenerative Diseases (DZNE), Göttingen, Germany. ${ }^{4}$ Laboratory of Pharmacology, School of Health Sciences, Department of Pharmacy, Aristotle University of Thessaloniki, Thessaloniki, Greece. ${ }^{5}$ German Center for Neurodegenerative Diseases (DZNE), Bonn, Germany. ${ }^{6}$ Bellvitge University Hospital-IDIBELL, Department of Pathology and Experimental Therapeutics, University of Barcelona, Hospitalet de Llobregat, Spain. 'European Neuroscience Institute, Göttingen, Germany. ${ }^{8}$ Institut National de la Recherche Agronomique/Ecole Nationale Vétérinaire, Toulouse, France. ${ }^{9}$ Centro de Investigación en Sanidad Animal (CISA-INIA), Madrid, Spain. ${ }^{10}$ Institute of Neuropathology, University Medical Center Hamburg-Eppendorf, Hamburg, Germany. ${ }^{11}$ Alzheimer's Disease and Other Cognitive Disorders Unit, Neurology Department, Hospital Clínic, Institut d'Investigacions
Biomediques August Pi i Sunyer (IDIBAPS), Barcelona, Spain. ${ }^{12}$ Present address: Unit of Lymphoid Malignancies, Division of Experimental Oncology, San Raffaele Scientific Institute, Milan, Italy.

Received: 22 June 2017 Accepted: 30 October 2017

Published online: 10 November 2017

\section{References}

1. Kazakova MH, Sarafian VS. YKL-40-a novel biomarker in clinical practice? Folia Med. 2009;51:5-14.

2. Renkema GH, Boot RG, Au FL, Donker-Koopman WE, Strijland A, Muijsers AO, et al. Chitotriosidase a chitinase, and the 39-kDa human cartilage glycoprotein, a chitin-binding lectin, are homologues of family 18 glycosyl hydrolases secreted by human macrophages. Eur J Biochem. 1998:251:504-9.

3. Schultz NA, Johansen JS. YKL-40-a protein in the field of translational medicine: A role as a biomarker in cancer patients? Cancers (Basel). 2010;2:1453-91.

4. Shao R. YKL-40 acts as an angiogenic factor to promote tumor angiogenesis. Front. Physiol. 2013;4:122.

5. Ringsholt M, Høgdall EVS, Johansen JS, Price PA, Christensen LH. YKL-40 protein expression in normal adult human tissues - an immunohistochemical study. J Mol Histol [lnternet] 2007;38:33-43. Available from: https://www.ncbi.nlm.nih.gov/pubmed/17242979, http://link.springer. com/10.1007/s10735-006-9075-0

6. Shao R, Hamel K, Petersen L, Cao QJ, Arenas RB, Bigelow C, et al. YKL-40, a secreted glycoprotein, promotes tumor angiogenesis. Oncogene. 2009;28: 4456-68. Available from: http://www.pubmedcentral.nih.gov/articlerender. fcgi?artid $=2795793 \&$ tool $=$ pmcentrez\&rendertype $=$ abstract

7. Rathcke CN, Vestergaard H. YKL-40-an emerging biomarker in cardiovascular disease and diabetes. Cardiovasc Diabetol. 2009:8:7.

8. Rehli M, Niller HH, Ammon C, Langmann S, Schwarzfischer L, Andreesen R, et al. Transcriptional regulation of CHI3L1, a marker gene for late stages of macrophage differentiation. J Biol Chem. 2003;278:44058-67.

9. Rehli M, Krause SW, Andreesen R. Molecular characterization of the gene for human cartilage gp-39 (CHI3L1), a member of the chitinase protein family and marker for late stages of macrophage differentiation. Genomics. 1997:43:221-5.

10. Qin G, Li X, Chen Z, Liao G, Su Y, Chen Y, et al. Prognostic value of YKL-40 in patients with Glioblastoma: a systematic review and meta-analysis. Mol Neurobiol. 2017;54:3264-70

11. Francescone RA, Scully S, Faibish M, Taylor SL, Oh D, Moral L, et al. Role of YKL-40 in the angiogenesis, radioresistance, and progression of glioblastoma. J Biol Chem. 2011;286:15332-43.

12. Xiang W, Menges S, Schlachetzki JC, Meixner H, Hoffmann A-C, SchlötzerSchrehardt $\mathrm{U}$, et al. Posttranslational modification and mutation of histidine 50 trigger alpha synuclein aggregation and toxicity. Mol. Neurodegener. 2015;10:8.[cited 2015 Aug 18]; Available from: http://www.molecularneurode generation.com/content/10/1/8

13. Johansen JS. Studies on serum YKL-40 as a biomarker in diseases with inflammation, tissue remodelling, fibroses and cancer. Dan Med Bull. 2006; 53:172-209.

14. Michelsen AE, Rathcke CN, Skjelland M, Holm S, Ranheim T, Krohg-Sorensen $\mathrm{K}$, et al. Increased YKL-40 expression in patients with carotid atherosclerosis. Atherosclerosis. 2010;211:589-95. Available from: http://ac.els-cdn.com/ S0021915010001760/1-s2.0-S0021915010001760-main.pdf?_tid=8d09dab477fd-1 1 e5-a89a-00000aab0f01 \&acdnat=1445436838_1a64d9b28138f5981ec f641d6062a3do

15. Bonneh-Barkay D, Bissel SJ, Wang G, Fish KN, Nicholl GCB, Darko SW, et al. YKL-40, a marker of simian immunodeficiency virus encephalitis, modulates the biological activity of basic fibroblast growth factor. Am J Pathol 2008; 173:130-43. Available from: http://www.pubmedcentral.nih.gov/ articlerender.fcgi?artid $=2438291 \&$ tool $=$ pmcentrez\&rendertype $=$ abstract.

16. Bonneh-Barkay D, Wang G, Starkey A, Hamilton RL, Wiley CA. In vivo CHI3L1 (YKL-40) expression in astrocytes in acute and chronic neurological diseases.J. Neuroinflammation. 2010;7:34. Available from: http://www.pubmedcentral.nih. gov/articlerender.fcgi?artid=2892443\&tool=pmcentrez\&rendertype=abstract.

17. Bonneh-Barkay D, Zagadailov P, Zou H, Niyonkuru C, Figley M, Starkey A, et al. YKL-40 expression in traumatic brain injury: an initial analysis. J Neurotrauma [Internet]. 2010;27:1215-23. Available from: http://www.pubmedcentral.nih.gov/ articlerender.fcgi?artid=2942903\&tool=pmcentrez\&rendertype $=$ abstract. 
18. Comabella M, Fernández M, Martin R, Rivera-Vallvé S, Borrás E, Chiva C, et al. Cerebrospinal fluid chitinase 3-like 1 levels are associated with conversion to multiple sclerosis. Brain. 2010;133:1082-93.

19. Johansen JS, Baslund B, Garbarsch C, Hansen M, Stoltenberg M, Lorenzen I, et al. YKL-40 in giant cells and macrophages from patients with giant cell arteritis. Arthritis Rheum [Internet]. 1999;42:2624-30. Available from: https:// www.ncbi.nlm.nih.gov/pubmed/10616010.

20. Junker $\mathrm{N}$, Johansen JS, Andersen CB, Kristjansen PE. Expression of YKL-40 by peritumoral macrophages in human small cell lung cancer. Lung Cancer [Internet]. 2005;48:223-31. Available from: http://www.ncbi.nlm.nih.gov/ entrez/query.fcgi?cmd=Retrieve\&db=PubMed\&dopt=Citation\&list_uids= 15829322.

21. Létuvé S, Kozhich A, Arouche N, Grandsaigne M, Reed J, Dombret M, et al. YKL-40 is elevated in patients with chronic obstructive pulmonary disease and activates alveolar macrophages. J Immunol. 2008;181:5167-73.

22. Bonneh-Barkay D, Bissel SJ, Kofler J, Starkey A, Wang G, Wiley CA. Astrocyte and macrophage regulation of YKL-40 expression and cellular response in neuroinflammation. Brain Pathol. 2012;22:530-46.

23. Craig-Schapiro R, Perrin RJ, Roe CM, Xiong C, Carter D, Cairns NJ, et al. YKL-40: a novel prognostic fluid biomarker for preclinical Alzheimer's disease. Biol Psychiatry. 2010;68:903-12.

24. Olsson B, Hertze J, Lautner R, Zetterberg H, Nägga K, Höglund K, et al. Microglial markers are elevated in the prodromal phase of Alzheimer's disease and vascular dementia. J Alzheimers Dis. 2013;33:45-53.

25. Antonell A, Mansilla A, Rami L, Lladó A, Iranzo A, Olives J, et al. Cerebrospinal fluid level of YKL-40 protein in preclinical and prodromal Alzheimer's disease. J Alzheimers Dis. 2014;42:901-8.

26. Kester MI, Teunissen CE, Sutphen C, Herries EM, Ladenson JH, Xiong CJ, et al. Cerebrospinal fluid VILIP-1 and YKL-40, candidate biomarkers to diagnose, predict and monitor Alzheimer's disease in a memory clinic cohort. Alzheimers Res Ther. 2015;7:9.

27. Verkhratsky A, Olabarria M, Noristani HN, Yeh CY, Rodriguez JJ. Astrocytes in Alzheimer's disease. Neurotherapeutics. 2010;7:399-412.

28. Gispert JD, Monté GC, Falcon C, Tucholka A, Rojas S, Sánchez-Valle R, et al. CSF YKL-40 and pTau181 are related to different cerebral morphometric patterns in early AD. Neurobiol Aging. 2016;38:47-55.

29. Perrin RJ, Craig-Schapiro R, Malone JP, Shah AR, Gilmore P, Davis AE, et al. Identification and validation of novel cerebrospinal fluid biomarkers for staging early Alzheimer's disease. PLoS One. 2011;6:e16032.

30. Wildsmith KR, Schauer SP, Smith AM, Arnott D, Zhu Y, Haznedar J, et al. Identification of longitudinally dynamic biomarkers in Alzheimer's disease cerebrospinal fluid by targeted proteomics. Mol. Neurodegener. 2014;9:22. Available from: http://www.ncbi.nlm.nih.gov/pubmed/24902845\%5Cn, http://www.pubmedcentral.nih.gov/articlerender.fcgi?artid=PMC4061120.

31. Rosen C, Andersson CH, Andreasson U, Molinuevo JL, Bjerke M, Rami L, et al. Increased Levels of Chitotriosidase and YKL-40 in Cerebrospinal Fluid from Patients with Alzheimer's Disease. Dement Geriatr Cogn Dis Extra. 2014;4:297-304. Available from: http://www.ncbi.nlm.nih.gov/pmc/articles/ PMC4164083/pdf/dee-0004-0297.pdf.

32. Wennström M, Surova $Y$, Hall S, Nilsson C, Minthon L, Hansson O, et al. The inflammatory marker YKL-40 is elevated in cerebrospinal fluid from patients with Alzheimer's but not Parkinson's disease or dementia with Lewy bodies. PLoS One. 2015;10:e0135458.

33. Paterson RW, Heywood WE, Heslegrave AJ, Magdalinou NK, Andreasson U, Sirka E, et al. A targeted proteomic multiplex CSF assay identifies increased malate dehydrogenase and other neurodegenerative biomarkers in individuals with Alzheimer's disease pathology. Transl. Psychiatry. 2016;6:e952. Available from: http://www.nature.com/doifinder/10.1038/tp.2016.194.

34. Alcolea D, Carmona-Iragui M, Suarez-Calvet M, Sanchez-Saudinos MB, Sala I, Anton-Aguirre S, et al. Relationship between beta-Secretase, inflammation and core cerebrospinal fluid biomarkers for Alzheimer's disease. J Alzheimers Dis. 2014;42:157-67. Available from: http://content.iospress.com/articles/ journal-of-alzheimers-disease/jad140240.

35. Janelidze S, Hertze J, Zetterberg H, Landqvist Waldo M, Santillo A, Blennow K, et al. Cerebrospinal fluid neurogranin and YKL-40 as biomarkers of Alzheimer's disease. Ann. Clin. Transl. Neurol. 2015;3 Available from: http://doi.wiley.com/10. 1002/acn3.266

36. Teunissen CE, Elias N, Koel-Simmelink MJA, Durieux-Lu S, Malekzadeh A, Pham TV, et al. Novel diagnostic cerebrospinal fluid biomarkers for pathologic subtypes of frontotemporal dementia identified by proteomics. Alzheimer's Dement. 2016;2:86-94.
37. Baldacci F, Toschi N, Lista S, Zetterberg H, Blennow K, Kilimann I, et al. Twolevel diagnostic classification using cerebrospinal fluid YKL-40 in Alzheimer's disease. Alzheimers. Dement. 2017:1-11. Available from: http://www. alzheimersanddementia.com/article/S1552-5260(17)30047-X/abstract. http:// www.ncbi.nlm.nih.gov/pubmed/28263742.

38. López González I, Garcia-Esparcia P, Llorens F, Ferrer I. Genetic and transcriptomic profiles of inflammation in neurodegenerative diseases: Alzheimer, Parkinson, Creutzfeldt-Jakob and Tauopathies. Int J Mol Sci. 2016;17:1-23.

39. Llorens F, Ansoleaga B, Garcia-Esparcia P, Zafar S, Grau-Rivera O, López-González I, et al. PrP mRNA and protein expression in brain and PrP(c) in CSF in CreutzfeldtJakob disease MM1 and W2. Prion. 2013;7:383-93. Available from: http://www. tandfonline.com/doi/abs/10.4161/pri.26416.

40. Llorens F, Lopez-Gonzalez I, Thune K, Carmona M, Zafar S, Andeoletti O, et al. Subtype and regional-specific neuroinflammation in sporadic creutzfeldt-jakob disease. Front Aging Neurosci. 2014;6:198.

41. Lopez-Gonzalez I, Schluter A, Aso E, Garcia-Esparcia P, Ansoleaga B, LL F, et al. Neuroinflammatory signals in Alzheimer disease and APP/PS1 transgenic mice: correlations with plaques, tangles, and Oligomeric species. J Neuropathol Exp Neurol. 2015;74:319-44. Available from: http://www.ncbi.n/m.nih.gov/pubmed/ 25756590

42. Garcia-Esparcia P, López-González I, Grau-Rivera O, García-Garrido MF, Konetti A, Llorens F, et al. Dementia with Lewy Bodies: Molecular Pathology in the Frontal Cortex in Typical and Rapidly Progressive Forms. Front. Neurol. 2017;8 Available from: http://journal.frontiersin.org/article/10.3389/fneur.2017.00089/full.

43. Puoti G, Bizzi A, Forloni G, Safar JG, Tagliavini F, Gambetti P. Sporadic human prion diseases: molecular insights and diagnosis. Lancet Neurol. 2012;11:618-28.

44. Llorens F, Schmitz M, Karch A, Cramm M, Lange P, Gherib K, et al. Comparative analysis of cerebrospinal fluid biomarkers in the differential diagnosis of neurodegenerative dementia. Alzheimers Dement. 2015:1-13.

45. Llorens F, Kruse N, Schmitz M, Gotzmann N, Golanska E, Thüne K, et al. Evaluation of a-synuclein as a novel cerebrospinal fluid biomarker in different forms of prion diseases. Alzheimer's Dement. 2016:1-10. Available from: http://linkinghub.elsevier.com/retrieve/pii/S1552526016330552.

46. Schmidt C, Wolff M, Weitz M, Bartlau T, Korth C, Zerr I. Rapidly progressive Alzheimer disease. Arch Neurol. 2011;68:1124-30.

47. Villemagne $V L$, Burnham S, Bourgeat P, Brown B, Ellis KA, Salvado O, et al. Amyloid beta deposition, neurodegeneration, and cognitive decline in sporadic Alzheimer's disease: a prospective cohort study. Lancet Neurol. 2013;12:357-67.

48. Roman G, Tatemichi T, Erkinjuntti T, Cummings J, Masdeu J, Garcia J, et al. Vascular dementia: diagnostic criteria for research studies: report of the NINDS-AIREN international workshop. Neurology. 1993;43:250-60.

49. McKhann G, Drachman D, Folstein M, Katzman R, Price D, Stadlan EM. Clinical diagnosis of Alzheimer's disease: report of the NINCDS-ADRDA work group* under the auspices of Department of Health and Human Services Task Force on Alzheimer's disease. Neurol Int. 1984;34:939. Available from: http://www. neurology.org/content/34/7/939.

50. Zerr I, Kallenberg K, Summers DM, Romero C, Taratuto A, Heinemann U, et al. Updated clinical diagnostic criteria for sporadic Creutzfeldt-Jakob disease. Brain. 2009;132:2659-68.

51. Zerr I, Pocchiari M, Collins S, Brandel JP, de Pedro Cuesta J, Knight RS, et al. Analysis of EEG and CSF 14-3-3 proteins as aids to the diagnosis of Creutzfeldt-Jakob disease. Neurology. 2000;55:811-5. Available from: https://www.ncbi.nlm.nih.gov/pubmed/10994001.

52. Padilla $D$, Béringue $V$, Espinosa JC, Andreoletti $O$, Jaumain $E$, Reine $F$, et al. Sheep and goat BSE propagate more efficiently than cattle BSE in human PrP transgenic mice. PLoS Pathog. 2011;7(3):e1001319.

53. Oakley H, Cole SL, Logan S, Maus E, Shao P, Craft J, et al. Intraneuronal $\beta$ amyloid aggregates, Neurodegeneration, and neuron loss in transgenic mice with five familial Alzheimer's disease mutations: potential factors in amyloid plaque formation. J Neurosci [Internet]. 2006;26:10129-40. Available from: http://www.jneurosci.org/content/26/40/10129.abstract.

54. Eimer WA, Vassar R. Neuron loss in the 5XFAD mouse model of Alzheimer's disease correlates with intraneuronal AB42 accumulation and Caspase-3 activation. Mol. Neurodegener. 2013;8:2. Available from: http://www.ncbi. nlm.nih.gov/pubmed/23316765\%5Cn, http://www.pubmedcentral.nih.gov/ articlerender.fcgi?artid=PMC3552866.

55. Wolf $\mathrm{H}$, Hossinger A, Fehlinger A, Büttner $\mathrm{S}$, Sim V, McKenzie $\mathrm{D}$, et al Deposition pattern and subcellular distribution of disease-associated prion protein in cerebellar organotypic slice cultures infected with scrapie. Front Neurosci. 2015;9:410. 
56. Zerr I, Bodemer M, Gefeller O, Otto M, Poser S, Wiltfang J, et al. Detection of 14-3-3 protein in the cerebrospinal fluid supports the diagnosis of CreutzfeldtJakob disease. Ann Neurol. 1998;43:32-40.

57. Milosevic I, Giovedi S, Lou X, Raimondi A, Collesi C, Shen H, et al. Recruitment of endophilin to clathrin-coated pit necks is required for efficient vesicle uncoating after fission. Neuron. 2011;72:587-601.

58. Schmitz M, Cramm M, Llorens F, Collins S, Atarashi R, Satoh K, et al. The realtime quaking-induced conversion assay for detection of human prion disease and study of other protein misfolding diseases. Nat Protoc. 2016;11:2233-42.

59. Cramm M, Schmitz M, Karch A, Mitrova E, Kuhn F, Schroeder B, et al. Stability and reproducibility underscore utility of RT-QulC for diagnosis of CreutzfeldtJakob disease. Mol Neurobiol. 2016;53:1896-904.

60. Youden WJ. Index for rating diagnostic tests. Cancer. 1950;3:32-5.

61. Windl O, Giese A, Schulz-Schaeffer W, Zerr I, Skworc K, Arendt S, et al. Molecular genetics of human prion diseases in Germany. Hum Genet. 1999;105:244-52.

62. Falsig J, Sonati T, Herrmann US, Saban D, Li B, Arroyo K, et al. Prion pathogenesis is faithfully reproduced in cerebellar Organotypic slice cultures. PLoS Pathog. 2012;8(11):e1002985.

63. Hill AF. Molecular classification of sporadic Creutzfeldt-Jakob disease. Brain. 2003;126:1333-46. Available from: http://brain.oxfordjournals.org/cgi/content/ abstract/126/6/1333.

64. Heinemann U, Krasnianski A, Meissner B, Varges D, Kallenberg K, SchulzSchaeffer WJ, et al. Creutzfeldt-Jakob disease in Germany: a prospective 12-year surveillance. Brain. 2007;130:1350-9.

65. Heneka MT, Carson MJ, El KJ, Landreth GE, Brosseron F, Feinstein DL, et al. Neuroinflammation in Alzheimer's disease. Lancet Neurol. 2015; 14(4):388-405.

66. Minter MR, Taylor JM, Crack PJ. The contribution of neuroinflammation to amyloid toxicity in Alzheimer's disease. J. Neurochem. 2016;136(3):457-74.

67. Radford RA, Morsch M, Rayner SL, Cole NJ, Pountney DL, Chung RS. The established and emerging roles of astrocytes and microglia in amyotrophic lateral sclerosis and frontotemporal dementia. Front. Cell. Neurosci. 2015;9: 414. Available from: http://www.pubmedcentral.nih.gov/articlerender.fcgi?artid= 4621294\&tool=pmcentrez\&rendertype=abstract.

68. Ugbode C, Hu Y, Whalley B, Peers C, Rattray M, Dallas ML. Astrocytic transporters in Alzheimer's disease. Biochem J [Internet]. 2017;474:333-55. Available from: http://biochemj.org/lookup/doi/10.1042/BCJ20160505.

69. Simpson JE, Ince PG, Lace G, Forster G, Shaw PJ, Matthews F, et al. Astrocyte phenotype in relation to Alzheimer-type pathology in the ageing brain. Neurobiol Aging. 2010;31:578-90.

70. Fuller S, Steele M, Münch G. Activated astroglia during chronic inflammation in Alzheimer's disease-Do they neglect their neurosupportive roles? Mutat. Res. 2010;690(1-2):40-9.

71. Donato R, Sorci G, Riuzzi F, Arcuri C, Bianchi R, Brozzi F, et al. S100B's double life: Intracellular regulator and extracellular signal. Biochim. Biophys. Acta. 2009;1793(6):1008-22.

72. Van Eldik LJ, Wainwright MS. The Janus face of glial-derived S100B: beneficial and detrimental functions in the brain. Restor. Neurol. Neurosci. 2003;21:97108. Available from: http://www.ncbi.nlm.nih.gov/pubmed/14530573.

73. Böhmer AE, Oses JP, Schmidt AP, Perón CS, Krebs CL, Oppitz PP, et al, Neuron-specific enolase, S100B, and glial fibrillary acidic protein levels as outcome predictors in patients with severe traumatic brain injury. Neurosurgery. 2011;68:1624-30.

74. Petzold A, Jenkins R, Watt HC, Green AJE, Thompson EJ, Keir G, et al. Cerebrospinal fluid S100B correlates with brain atrophy in Alzheimer's disease. Neurosci Lett. 2003:336:167-70.

75. Llorens F, Schmitz M, Knipper T, Schmidt C, Lange P, Fischer A, et al. Cerebrospinal Fluid Biomarkers of Alzheimer's Disease Show Different but Partially Overlapping Profile Compared to Vascular Dementia. Front. Aging Neurosci. 2017;9:1-9. Available from: http://journal.frontiersin.org/article/10. 3389/fnagi.2017.00289/full.

76. Sanchez-Juan P, Green A, Ladogana A, Cuadrado-Corrales N, Sáanchez-Valle R, Mitrováa E, et al. CSF tests in the differential diagnosis of CreutzfeldtJakob disease. Neurology. 2006;67:637-43.

77. Fuller S, Munch G, Steele M. Activated astrocytes: a therapeutic target in Alzheimer's disease? Expert rev Neurother. 2009;9:1585-94. Available from: http://www.ncbi.nlm.nih.gov/pubmed/19903019.

78. Wiley CA, Bonneh-Barkay D, Dixon CE, Lesniak A, Wang G, Bissel SJ, et al. Role for mammalian chitinase 3-like protein 1 in traumatic brain injury. Neuropathology. 2015;35:95-106.
79. Bonneh-Barkay D, Wang GJ, LaFramboise WA, Wiley CA, Bissel SJ. Exacerbation of experimental autoimmune encephalomyelitis in the absence of breast regression protein 39/Chitinase 3-like 1. J Neuropathol Exp Neurol. 2012;71:948-58.

80. Dalgard CL, Cole JT, Kean WS, Lucky JJ, Sukumar G, McMullen DC, et al. The cytokine temporal profile in rat cortex after controlled cortical impact. Front Mol Neurosci. 2012;5:6.

81. Bhardwaj R, Yester JW, Singh SK, Biswas DD, Surace MJ, Waters MR, et al. RelB/p50 complexes regulate cytokine-induced YKL-40 expression. J Immunol. 2015;194:2862-70.

82. Liddelow SA, Guttenplan KA, Clarke LE, Bennett FC, Bohlen CJ, Schirmer L, et al. Neurotoxic reactive astrocytes are induced by activated microglia. Nature. 2017; Available from: http://www.nature.com/doifinder/10.1038/ nature21029.

83. Lian H, Litvinchuk A, Chiang AC-A, Aithmitti N, Jankowsky JL, Zheng H. Astrocyte-microglia cross talk through complement activation modulates amyloid pathology in mouse models of Alzheimer's disease. J. Neurosci, Available from. 2016;36:577-89. http://www.ncbinlm.nih.gov/pubmed/26758846.

84. Schwab C, McGeer PL. Inflammatory aspects of Alzheimer disease and other neurodegenerative disorders. J Alzheimers Dis. 2008;13:359-69.

85. Shepherd CE, Thiel E, McCann H, Harding AJ, Halliday GM. Cortical inflammation in Alzheimer disease but not dementia with Lewy bodies. Arch. Neurol. 2000;57: $817-22$

86. Llorens F, Kruse N, Karch A, Schmitz M, Zafar S, Gotzmann N, et al. Validation of a-Synuclein as a CSF biomarker for sporadic Creutzfeldt-Jakob disease. Mol. Neurobiol. 2017:1-9. Available from: http://link.springer.com/10.1007/s12035017-0479-5.

87. Stoeck K, Schmitz M, Ebert E, Schmidt C, Zerr I. Immune responses in rapidly progressive dementia: a comparative study of neuroinflammatory markers in Creutzfeldt-Jakob disease, Alzheimer is disease and multiple sclerosis. J. Neuroinflammation, Available from. 2014;11:170. http://www.ncbi.nlm.nih. gov/pubmed/25315814.

88. Llorens F, Karch A, Golanska E, Schmitz M, Lange P, Sikorska B, et al. Cerebrospinal Fluid Biomarker-Based Diagnosis of Sporadic CreutzfeldtJakob Disease: A Validation Study for Previously Established Cutoffs. Dement. Geriatr. Cogn. Disord. 2017;43:71-80. Available from: https:// www.karger.com/?doi=10.1159/000454802.

89. Hellwig K, Kvartsberg H, Portelius E, Andreasson U, Oberstein TJ, Lewczuk P, et al. Neurogranin and YKL-40: independent markers of synaptic degeneration and neuroinflammation in Alzheimer's disease. Alzheimers. Res. Ther. 2015;7:74. Available from: http://www.pubmedcentral.nih.gov/articlerender.fcgi?artid= 4690296\&tool=pmcentrez\&rendertype=abstract.

90. Mattsson N, Tabatabaei S, Johansson P, Hansson O, Andreasson U, Månsson JE, et al. Cerebrospinal fluid microglial markers in Alzheimer's disease: elevated chitotriosidase activity but lack of diagnostic utility. NeuroMolecular Med. 2011; 13:151-9.

91. Carter SF, Scholl M, Almkvist O, Wall A, Engler H, Langstrom B, et al. Evidence for Astrocytosis in Prodromal Alzheimer Disease Provided by 11C-DeuteriumL-Deprenyl: A Multitracer PET Paradigm Combining 11C-Pittsburgh Compound B and 18F-FDG. J. Nucl. Med. 2012;53:37-46.

92. Llorens F, Thüne K, Sikorska B, Schmitz M, Tahir W, Fernández-borges N. Altered Ca 2 + homeostasis induces Calpain- Cathepsin axis activation in sporadic Creutzfeldt-Jakob disease. Acta Neuropathol Commun. 2017;5(1):35.

93. Gispert JD, Monté GC, Suárez-Calvet M, Falcon C, Tucholka A, Rojas S, et al. The $\{A P O E\} \varepsilon 4$ genotype modulates $\{C S F\}$ YKL-40 levels and their structural brain correlates in the continuum of Alzheimer disease but not those of sTREM2. Alzheimer's Dement. Diagnosis, Assess. Dis. Monit. 2016;6 Available from: http://www.sciencedirect.com/science/article/pii/S2352872916300653.

94. Llorens F, Thune K, Schmitz M, Ansoleaga B, Frau-Mendez MA, Cramm M, et al. Identification of new molecular alterations in fatal familial insomnia. Hum Mol Genet. 2016;0:1-20.

95. Parchi P, Castellani R, Cortelli P, Montagna P, Chen SG, Petersen RB, et al. Regional distribution of protease-resistant prion protein in fatal familial insomnia. Ann Neurol. 1995;38:21-9.

96. Ladogana A, Sanchez-Juan P, Mitrova E, Green A, Cuadrado-Corrales N, Sanchez-Valle $R$, et al. Cerebrospinal fluid biomarkers in human genetic transmissible spongiform encephalopathies. J Neurol, Available from. 2009;256:1620-8. http://www.ncbi.nlm.nih.gov/pubmed/19444528.

97. Kovács GG, Puopolo M, Ladogana A, Pocchiari M, Budka H, van Duijn C, et al. Genetic prion disease: the EUROCJD experience. Hum Genet. 2005;1 18:166-74.

98. Gambetti P, Kong Q, Zou W, Parchi P, Chen SG. Sporadic and familial CJD: Classification and characterisation. Br. Med. Bull. 2003;66:213-39. 
99. Shahim P, Portelius E, Brody DL. Astroglial activation and altered amyloid metabolism in human repetitive concussion. 2017;

100. Hjalmarsson C, Bjerke M, Andersson B, Blennow K, Zetterberg H, Aberg ND, et al. Neuronal and glia-related biomarkers in cerebrospinal fluid of patients with acute ischemic stroke. J. Cent. Nerv. Syst. Dis. 2014;6:51-8. Available from: http://www.pubmedcentral.nih.gov/articlerender.fcgi?artid=4039292\&tool= pmcentrez\&rendertype $=$ abstract

101. Rymo I, Kern S, Bjerke M, Zetterberg H, Marlow T, Blennow K, et al. CSF YKL40 and GAP-43 are related to suicidal ideation in older women. Acta Psychiatr. Scand. 2017:351-7. Available from: http://doi.wiley.com/10.1111/acps.12701.

102. Jakobsson J, Bjerke M, Sahebi S, Isgren A, Ekman CJ, Sellgren C, et al. Monocyte and microglial activation in patients with mood-stabilized bipolar disorder. J Psychiatry Neurosci. 2015;40:250-8.

103. Van Everbroeck B, Dewulf E, Pals P, Lübke U, Martin JJ, Cras P. The role of cytokines, astrocytes, microglia and apoptosis in Creutzfeldt-Jakob disease. Neurobiol Aging. 2002;23:59-64.

Submit your next manuscript to BioMed Central and we will help you at every step:

- We accept pre-submission inquiries

- Our selector tool helps you to find the most relevant journal

- We provide round the clock customer support

- Convenient online submission

- Thorough peer review

- Inclusion in PubMed and all major indexing services

- Maximum visibility for your research

Submit your manuscript at www.biomedcentral.com/submit
Biomed Central 